\title{
Zeitlichkeit im bewegten Bild. Stillgestellte Körper zwischen Performanz und Pose
}

In den nun folgenden Abschnitten wird es darum gehen, die Korrespondenz zeitlicher und geschichtlicher Phänomene in konkreten Beispielen der Gegenwartskunst zu verdeutlichen. Hier tritt die Bewegung als formalästhetisches Charakteristikum des Bildes in den Vordergrund. Im Vergleich mehrerer Werke von James Coleman und Tacita Dean, in denen Figuren im Bild Posen wählen, um eine Strukturierung der Zeit vorzunehmen, werden Aspekte der Körperlichkeit beschrieben und der unterschiedliche Umgang mit dem posierenden Körper und seiner Repräsentation mittels Video, Fotografie und Film dargestellt. Die Bewegung als zeitliche Struktur am Bild zu untersuchen erlaubt es, unterschiedliche Phänomene als Formen der Manifestation und Darstellung von Zeit zu betrachten. Mit der Pose steht ein Motiv im Fokus, an dem sich die veränderliche Zeitlichkeit des Bildes in unterschiedlichen Medien konkretisieren und beschreiben lässt und dessen historische Bezüge die zeitgenössischen Werke stark beeinflussen.

Zwei Beispiele dienen primär der Anschauung: zunächst James Colemans Dia- und Videoarbeiten, in denen das Posieren von Personen eines der auffälligsten Merkmale ist; ihre Posen, ihre seltsame, spezifische Art, sich in ihrer Körperlichkeit zur Kamera zu verhalten, ist sehr typisch für Colemans Arbeiten der 198oer und 199oer Jahre. Was haben diese statischen, kühlen Posen zu bedeuten? Welche Mechanismen der Darstellung, der bildlichen Konventionen und der historischen Rezeptionszusammenhänge zitieren sie, wie und wieso wird beispielsweise auf die historische Kunstform des Tableau vivant verwiesen? Die Körper erfahren eine Stillstellung, die im Widerspruch zum durch und durch mobilen Medium der Diaprojektion bei Coleman steht. Sie unterscheiden sich maßgeblich von vorherrschenden Thematisierungen des Körpers in der Performancekunst und Body-Art, die mit dem klassischen Körperbild brechen wollen. Die Pose verbindet als übergeordnetes Gestaltungsprinzip die verschiedenen Medien Tanz, Fotografie und Film; sie betrifft einen >Ausnahmezustand < des Körpers, nämlich seine Verbindung zum Bildlichen und zum Stillstand anstatt der Bewegung. Darüber hinaus thematisiert die Pose das Verhältnis der fotografierten oder gefilmten Person zum Betrachter / zur Betrachterin, die Subjekt-Objekt-Beziehung und die Blickkonstellationen zwischen Posierenden und Betrachtenden. Sie adressiert Fragen der Identität 
und Selbstwahrnehmung und findet sich so auch in reflexionsphilosophischen Überlegungen wieder. ${ }^{1}$

Colemans Arbeiten sind bereits in zahlreichen Aufsätzen und Essays betrachtet worden; die Posen seiner Figuren, der Einsatz von Schauspielern und ihre spezielle Darstellung wurden dabei nicht gesondert untersucht, sondern stets in die Beschreibung der Medialität, der Narration und der Historizität seiner Werke eingegliedert. Von der Frage nach der Korrelation zwischen Temporalität und Historizität des Bildes ausgehend soll das Augenmerk auf diese Figuren gerichtet werden. Sie mit Blick auf die Pose als Figuration der Bewegung zu betrachten scheint zielführend, denn die Pose weist als Bildsujet eine strukturelle Ähnlichkeit zur Ästhetik der aktuellen Filmkünste auf, nämlich eine Stillstellung IN DER Bewegung. Den Arbeiten von Coleman, die hauptsächlich auf Fotografie beruhen, wird ein einzelnes Werk von Tacita Dean gegenübergestellt. In diesem porträtiert die Künstlerin den Tänzer und Choreografen Merce Cunningham, der für ihre Kamera agiert und dabei in zeitlicher Korrespondenz zum Aufbau des Werks 4'33" von John Cage verschiedene Posen einnimmt. Kann man bei Coleman die Pose in Bezug zur Fototheorie und einer »Archäologie der Medien $\aleph^{2}$ diskutieren, so steht bei Dean der Dialog zwischen Film, Tanz und Musik im Mittelpunkt. Die Pose als Schnittstelle zwischen den Künsten fungiert dabei auch als Mittlerin zwischen der Temporalität und Historizität der verschiedenen Bilder und Medien. Die aleatorische Zeitstruktur der Komposition überträgt sich mittels der Pose auf die filmische Installation; zugleich wird die Person des Tänzers, dessen gealterter Körper sich dem Tanz verweigert, in ihrer Unbeweglichkeit und Verletzlichkeit ausgestellt.

\section{Zeitlichkeit der Pose in Video und Diaprojektion bei James Coleman}

Die Videoarbeit So Different ... and Yet (1978-80) ist für James Coleman untypisch, da er ansonsten mit anderen Medien, vorwiegend Diaprojektion und Film, arbeitet. Es handelt sich um ein ca. 50-minütiges Video, in dem in einer geschlossenen Szenerie, einer Art Bühne, zwei Schauspieler auftreten: ein Mann am Flügel und eine Frau im Vordergrund auf einer Art Chaiselongue. Der

1 Vgl. die fototheoretische Untersuchung von Arndtz, Philosophie der Fotografie, besonders S. $15^{2-177}$.

2 Vgl. unter anderem Siegfried Zielinski, Archäologie der Medien. Zur Tiefenzeit des technischen Hörens und Sehens, Reinbek bei Hamburg 2002. 
männliche Schauspieler, Roger Doyle, trägt einen weißen Anzug und Hörner auf der Stirn und spielt leichte, improvisiert wirkende Kaffeehausmusik, die Frau, Olwen Fouéré, räkelt sich auf der Liege und trägt ein grün schimmerndes, kurzes Satinkleid mit paillettenbesetzten Spaghettiträgern, weiße lange Handschuhe und eine weiße Strumpfhose, wobei ein Bein mit einem roten Band umwickelt ist. Fouéré erzählt während des Videos eine Geschichte in die Kamera, ihre Sprechweise ist dabei sehr exaltiert und imitiert die Intonation einer Hörspielerzählerin, die mit verschieden starkem französischem Akzent die Rahmenhandlung sowie die weiblichen Rollen spricht. Die Story changiert zwischen einer Romanze und einer Detektivgeschichte, in der verschiedene Charaktere immer wieder auftreten; dabei übernimmt Doyle aus dem Hintergrund die männlichen Parts der Geschichte. Die Schauspielerin begleitet ihre Erzählung durch einen ständigen Wechsel ihrer Haltung in verschiedene Posen zwischen Sitzen und Liegen, ähnlich einem Model bei einem Fotoshooting, das sich immer wieder neu zur Kamera präsentieren und positionieren muss. Bisherige Interpretationen des Werks heben vor allem die Undurchdringlichkeit des narrativen Stoffs hervor, der, wie Jean Fisher es ausdrückt, fast implodiert: »[T]he plot/s never cease to thicken and narrative closure is deferred. Like the serialised soap opera, the story is forever >to be continued .... « ${ }^{3}$ Zahlreiche Personen werden namentlich vorgestellt und ihr Zusammentreffen in einem dress shop und auf einer Dinnerparty bei einem Paar namens Clayton beschrieben, wobei in Rückblicken die persönlichen Beziehungen der Akteure Norman, Clarissa, Vanna, Laura, Betty und anderen zueinander skizziert, aber keineswegs erhellt werden. Wie Linda Schädler beschreibt, werden zwei Handlungsstränge miteinander verwoben, zum einen die Vorbereitung und die Ereignisse auf der Dinnerparty der Claytons, zum anderen die Geschichte eines Raubüberfalls, in dessen Nachgang ein Einbruch und eine Entführung stattfinden, die aber nicht aufgeklärt werden. ${ }^{4}$

3 Jean Fisher, »So Different ... and Yet«, in: Irish Museum of Modern Art, Projects Arts Centre, Royal Hibernian Academy of Arts (Hg.), James Coleman, Ausstellungskatalog Irish Museum of Modern Art, Projects Arts Centre, Royal Hibernian Academy of Arts, Dublin 20og, S. 35-47, S. 40.

4 Eine bestmögliche Zusammenfassung der Ereignisse bietet Schädler, James Coleman und die Anamorphose, S. 95-99. Sie beschreibt auch zutreffend, dass es sich nicht um eine einfache Verbindung zweier klar voneinander zu trennender Erzählstränge handelt, sondern die Erzähllogik fundamental gebrochen wird, da die Identität der Personen unklar bleibt, sie zum Teil in beide Geschichten verwickelt sind, spontan ihre Handlungsweise ändern oder mit anderen Personen verwechselt werden. Der Zuschauer / die Zuschauerin sieht sich immer wieder neu vor der Herausforderung, das Personal der Geschichte(n) konsistenten Handlungen zuzuordnen, und muss ob der Fülle der nur angedeuteten Informationen zwangsläufig scheitern. Schädler interpretiert die Verknüpfung der beiden Genres Melodram 
Die Arbeit, welche ab 1978 konzipiert und schließlich nach monatelangen Proben 1980 mit den beiden Schauspielern in Dublin aufgenommen wurde, durchlief hinsichtlich ihrer Präsentationsform zahlreiche Transformationen. Ursprünglich wurde das Video auf einem Fernseher gezeigt, der Zuschauer oder die Zuschauerin saß ähnlich wie die Hauptdarstellerin auf einem Sofa in einem grün beleuchteten Raum. Später konnte So Different ... and Yet auf einem Fernseher, jedoch von einem größeren Publikum auf Stuhlreihen gesehen werden; 2007 war die Arbeit als Videoprojektion installiert, 2009 schließlich auf einem großen, freistehenden LED-Screen im Innenhof des Museum of Modern Art in Dublin. Das Video erscheint den Betrachter/innen als einzelne Aufnahme ohne Schnitte, tatsächlich sind jedoch drei Aufnahmen aneinandermontiert worden, und der Bildausschnitt wird zeitweise durch leichte Zooms und Schwenks verändert. Immer bleibt jedoch die im Vordergrund agierende Frau im Bildmittelpunkt. Coleman nutzte für die visuellen Effekte die Technik des Chroma Key, ein Verfahren, das eigentlich dazu gedacht ist, Personen im Nachhinein vor beliebige Hintergründe zu setzen, damit aber auch ermöglicht, verschiedene Aufnahmen nachträglich ineinanderzufügen. In der Präsentation erscheinen die durch Chroma Key retuschierten Bereiche in einem leuchtenden, fluoreszierenden Blau, welches die Zwischenräume zwischen den Figuren unwirklich und flach erscheinen lässt und im Widerspruch zu dem in der Inszenierung erzeugten Bühnencharakter steht. Jean Fisher hat dies so interpretiert, dass dadurch offengelassen werde, ob die beiden Personen tatsächlich im selben Raum und in derselben Zeit agierten. ${ }^{5}$

Bei der Deutung der Arbeit standen bisher vor allem drei Aspekte im Vordergrund: zunächst Colemans Reverenzen an Genres der Unterhaltungskultur wie das Melodram, die Romanze und den Krimi, an Exempel der low culture in Literatur und Fernsehen, die die Erzählweise und den Inhalt der Geschichten prägen; ${ }^{6}$ dann die bereits beschriebene Verschachtelung der Erzählung und die dadurch entstehende Notwendigkeit der aktiven Rezeption durch die Zuschauer/innen; ${ }^{7}$ schließlich die visuellen Verweise auf kunsthistorische

und Krimi als anamorphotisch, wobei Norman als Scharnierstelle der Kippfigur erscheine, da er in beiden Geschichten zentrale Rollen einnehme (vgl. ebd., S. 101).

5 Fisher, »So Different ... and Yet«, S. 38.

6 Fisher, »So Different ... and Yet«; Jean-Christophe Royoux, »Expanded Spectatorship. Narrative Strategies in the Work of James Coleman«, in: Fundació Antoni Tàpies (Hg.), James Coleman, Ausstellungskatalog Fundació Antoni Tàpies, Barcelona 1999/200o, Barcelona 1999, S. 27-49; Maeve Connolly, »Staging Television. James Coleman’s So Different ... and Yet«, in: Mousse Magazine 27, Februar 2011, S. 160-165.

7 Schädler,James Coleman und die Anamorphose. 
Vorbilder wie Manets Olympia oder die weibliche Odaliske, wobei die Rolle des weiblichen Körpers als Objekt des männlichen Blicks betont wird. ${ }^{8}$

An letzteren Punkt anschließend wird sich die folgende Besprechung auf die Figuren in Colemans Werk konzentrieren, ihre tableauhafte Darstellung, Blicke und Posen. Neben den unwirklichen Settings, der Farbigkeit der Bilder und der jeweils speziellen Modulation des Voiceovers stellen die meist statischen Figuren eines der herausstechenden Merkmale seiner Arbeiten dar. Mit Blick auf das Verhältnis von Zeitlichkeit und Geschichtlichkeit im Werk von James Coleman können folgende Aspekte zueinander in Beziehung gesetzt werden: die Zeitlichkeit der Pose und des Narrativs (Melodram) sowie die Zeitlichkeit des fotografisch festgehaltenen Körpers und die Geschichtlichkeit der Medien, die hier als Folien der Darstellung dienen: Fernsehen (mit Serie und Fotonovela), Malerei und Theater.

\section{Tableau vivant}

James Colemans Videoarbeit So Different ... and Yet und seine Diaprojektionen der 1980er und 199oer Jahre rufen unmittelbar Assoziationen zum historischen Phänomen des Tableau vivant hervor, wobei es neben einigen Ähnlichkeiten jedoch auch starke Differenzen gibt. Man kann das Tableau vivant als rein historisches Phänomen betrachten, das seine Blütezeit im späten 18. und im 19. Jahrhundert hatte. Als großbürgerliche Kunstform der Goethezeit, die zwischen Malerei, Plastik und Theater anzusiedeln ist, ist es vor allem durch literarische Beschreibungen wie in Goethes Wahlverwandtschaften (1809) oder später in Edith Whartons The House of Mirth (1905) überliefert. Das Tableau vivant, also das Nachstellen mythischer oder religiöser Szenen oder bekannter Gemälde zum Beispiel von Leonardo da Vinci, Nicolas Poussin oder Sandro Botticelli, diente als gesellschaftlicher Zeitvertreib, der auf der Seite der Darsteller/innen schauspielerisches Talent, Körperbeherrschung und Ausstattung der Szenerie, auf der Seite des Publikums Kunstkennerschaft und Einfühlung voraussetzt. ${ }^{9}$ Die zeitgleich auftauchende Attitüde war eine rein weibliche Körperkunst, die ohne große Dekoraktion auskam und einzelne Damen wie

8 Buchloh, »James Coleman«; Michael Newman, »Allegories of the Subject. The Theme of Identity in the Work of James Coleman «, in:James Coleman - selected works, Ausstellungskatalog The Renaissance Society at The University of Chicago, Institute of Contemporary Arts, London, 1985/1986, London 1985, S. 26-53, S. 31.

9 Zur Geschichte des Tableau vivant vgl. Sabine Folie, Michael Glasmeier, »Atmende Bilder. Tableau vivant und Attitüde zwischen >Wirklichkeit und Imagination « Tableaux vivants. Lebende Bilder und Attitüden in Fotografie, Film und Video, Ausstellungskatalog Kunsthalle Wien, Wien 2002, S. 9-52. 
Lady Emma Hamilton große Berühmtheit erlangen ließ. ${ }^{10}$ Im Gegensatz zu den Tableaux vivants, bei denen es sich stets um Gruppenszenen handelte, die mehrere Minuten gehalten wurden und in denen die kunsthistorischen Referenzen im Vordergrund standen, wurden Künstlerinnen der Attitüde durch ihre rasch wechselnden ausdrucksvollen und exaltierten Posen bekannt. Über die Untersuchung als historisches Phänomen hinaus ist das Tableau vivant auch im Hinblick auf sein Fortleben in Fotografie, Film und Video interessant. Hierbei stehen die Darstellung des Lebendigen, das Changieren zwischen bildnerischen und darstellerischen Gattungen, der Wechsel zwischen Stillstand und Bewegung sowie die historischen Referenzen der Tableaux vivants Pate. Die Attitüden sehen Sabine Folie und Michael Glasmeier in der weiblichen, zum Teil feministischen Performancekunst seit den 1970er Jahren wiederkehren:

In der Attitüde der Goethezeit hat die heutige Performance von Frauen, etwa von Marina Abramović, VALIE EXPORT, Yayoi Kusama, Carolee Schneemann oder HANNAH WILKE ihren Ursprung. Diese Künstlerinnen radikalisieren das Medium Attitüde in Richtung totaler Entblößung, die intellektuell motiviert, das Voyeurhafte desavouiert. Aus dem künstlerischen Akt der historischen Attitüde erwachsen die impliziten Pathosformeln weiblicher Selbstbestimmung. ${ }^{11}$

Wie Benjamin H. D. Buchloh beschreibt, nimmt James Coleman in seinen Werken ab den 198oer Jahren Bezug auf die Form des Tableau vivant und seine Möglichkeiten, bildende und darstellende Kunst, Malerei und Performance in den Medien Video und Fotografie zu verbinden. Coleman leitet mit diesen Werken einen Umbruch in seiner Ästhetik und Technik ein. Sein Frühwerk, welches zur Zeit seines Studiums in Mailand entstand, steht unter dem Einfluss von und in kritischer Auseinandersetzung mit dem Modernismus. In den Werken Flash Piece (1970), Slide Piece (1974) und Playback of a Daydream (1974), deren Arrangements fast schon wahrnehmungspsychologischen Untersuchungen gleichen, beschäftigt er sich mit Prozessen der visuellen Wahrnehmung und der sprachlichen Interpretation. Anders als

10 Vgl. zur Attitüde u. a. Dagmar von Hoff, Helga Meise, »Tableaux vivants. Die Kunst- und Kultform der Attitüden und lebenden Bilder«, in: Renate Berger, Inge Stephan (Hg.), Weiblichkeit und Tod in der Literatur, Köln/Wien 1987, S. 69-87; Bettina Baumgärtel, »Die Attitüde und die Malerei. Paradox der stillen Bewegtheit in Synthese von Erfindung und Nachahmung «, in: Zeitschrift des Deutschen Vereins für Kunstwissenschaft 46, 1992, S. 21-43; Gabriele Brandstetter, »Attitüden und Posen. Figuration als Bild und als Performance «, in: Atsuko Onuki (Hg.), Figuration - Defiguration. Beiträge zur transkulturellen Forschung, München 2006, S. 163-173.

Folie, Glasmeier, »Atmende Bilder«, S. 27. 
Vertreter des Postminimalismus und Konzeptualismus, denen Coleman in der Folge kritisch begegnet, bezieht er jedoch schon früh eine zu der Zeit vernachlässigte Dimension des Theatralischen und Historischen in seine Arbeiten ein. Buchloh arbeitet heraus, dass sich Coleman gegen zwei vorherrschende Doxai wendet: zum einen gegen Michael Frieds Kritik der als theatralisch verstandenen phänomenologischen Dimension der Malerei, zum anderen gegen die textuelle Ästhetik der zeitgenössischen Konzeptkünstler/innen, die sich aus der analytischen Philosophie oder der strukturalen Linguistik ableitete. ${ }^{12}$ »Theatralik« bedeutet für Coleman zu diesem frühen Zeitpunkt bereits, dass die traditionelle Auffassung vom visuellen Objekt als einem integrierten und privilegierten Ort des (ästhetischen) Wissens und der Erfahrung ebenso aufgehoben wird, wie die Auffassung von einer spezifischen visuellen Autonomie. $\ll^{13}$ Die Sprache unterzieht Coleman keiner linguistischen Kritik, sondern er stellt die $»$ Dimensionen der phonetischen Artikulation des Subjekts und die Funktion der rhetorischen Figuration der Sprache $\ll^{14}$ in den Mittelpunkt seines Interesses.

Dass Coleman sich in seiner Videoarbeit So Different ... and Yet der übertrieben theatralischen, körperlichen und sprachlichen Artikulation des postmodernen Melodrams widmet, ist also kein Zufall, sondern die radikale Einforderung der Anerkennung kultureller Wirksamkeit performativer und sprachlicher Prozesse zu einem Zeitpunkt, als diese in der high culture marginalisiert wurden. Dass gerade das Tableau vivant beziehungsweise die Attitüde als performative Ausdrucksformen in dieser Werkperiode aufgegriffen werden, ist Ausweis seines zunehmend historischen Interesses an Medien der Repräsentation und des Spektakels. Mit So Different ... and Yet schlägt Coleman einen Bogen von der frühmodernen bürgerlichen Unterhaltungsform zur konsumorientierten Fernsehkultur und zeigt auf, wie sich innerhalb verschiedener historischer Kontexte Mechanismen der Affektion, Narration und Repräsentation wiederholen. Die Erzählerin in dem Video fungiert als bewegter Hintergrund verschiedenartiger >Folien< oder Schichten kultureller Repräsentationsformen. Ihre körperliche >Verhüllung< in ein glitzerndes kurzes Kleid ist zugleich die Zurschaustellung ihrer körperlichen Reize, welche durch die rote Umwindung des Beins noch betont wird. Ihre Posen wechselt sie mit jeder Wendung in der erzählten Geschichte. Es bleibt unklar, ob die Erzählung auf ihre Bewegungen reagiert oder die Bewegungen auf die Erzählung. Wie in Colemans späteren Diaarbeiten, in denen im Voiceover

12 Vgl. Buchloh, »James Coleman«, S. 82-85.

13 Ebd., S. 84.

14 Ebd. 
die Erzählung mehr und mehr einer poetischen, auf Klang, Intonation und Geräuschen basierenden Stimmführung weicht, ist schon hier eine Wertschätzung des Rhythmus zu bemerken. Der Rhythmus als zeitliche Struktur erfasst gleichermaßen das Narrativ wie auch die Bewegungen des Körpers, sie befinden sich nicht im Gleichklang, nehmen aber in ihren Tempi aufeinander Bezug. Das auffallende Kleid der Darstellerin, welches auch eine tragende Rolle in der Geschichte spielt, und ihre Identität (handelt es sich bei ihr um Clarissa?) sind mit dem Schauspiel eng verknüpft, verwoben, um bei textilen beziehungsweise textuellen Metaphern zu bleiben. Dot Tuer und Jean Fisher haben mit Blick auf die sexualisierte Präsentation von Fouéré auf die Rolle der Frau als fetischisiertes Objekt im Kino hingewiesen und Colemans Arbeit als Provokation feministischer Kritik und Filmtheorie verstanden: $\gg$ By the 198os, the image of a woman dressed in a slinky cocktail dress and posing seductively to the camera/viewer was a provocation to feminist critiques of the female image as a construction by and for the voyeuristic male gaze $\ll^{15}$ Heute, so Fisher, würden man die Posen eher als Parodie des Verhaltens sogenannter Celebrities verstehen, die in ihrer öffentlichen Zurschaustellung die mediale Sichtbarkeit als Selbstzweck zelebrieren.

\section{Die Zeitlichkeit der Pose}

Wenn man die Pose als besondere Figur der Zeitlichkeit des Bildes ins Auge fassen möchte, ist zunächst zu klären, was eigentlich eine Pose ist und welche temporalen und historischen Dimensionen sie aufweist. Diese Frage nach der Pose betrifft eine spezifische bildliche Konfiguration, die den Körper in einem Zwischenzustand zwischen Stillstand und Bewegung hält: »Der Körper wird durch das Innehalten in der Bewegung, in der Pose einen Moment lang zur Plastik, zum Bild. Und er ist solchermaßen im Rahmen einer Bewegung, die anhält.« ${ }^{16}$ Das Verhältnis des posierenden Körpers zum Bild in der Pose kann über die besondere Zeitlichkeit, welche in der Bewegung und dem Innehalten sichtbar wird, beschrieben werden. Diese zeigt sich einerseits als »Zeitfigur choreografischer Komposition und des tänzerischen Ausdrucks« im Verhältnis zur Gebärde (Imdahl) und zur Attitüde, andererseits als »Verschiebung des Selbst« und »Mortifizierung « unter dem Blick des Anderen (Owens, Silverman) insbesondere in der Fotografie, schließlich als Zitat und als »Relais

15 Fisher, »So Different ... and Yet«, S. 40; Dot Tuer, »Feminist Pleasure in the Politics of Seduction «, in: C-magazine 4, 1985, S. 22-23.

16 Gabriele Brandstetter, Bettina Brandl-Risi, Stefanie Diekmann, »Posing Problems. Eine Einleitung«, in: dies. (Hg.), Hold it! Zur Pose zwischen Bild und Performance, Berlin 2012, S. $7-21$, S. 7 . 
zwischen den Künsten «. ${ }^{17}$ Dass die Pose durch ihr »Sowohl-als-auch von Stillstellung und Bewegung «18 als Mittlerin zwischen »Bild und Bildlichkeit« einerseits und »Performance und Performativität« andererseits geeignet sei, ist eine zentrale These von Gabriele Brandstetter, die die Pose in ebendiesem Spannungsfeld zwischen »picture « und »performance« sieht: »So gesehen ist die Pose, als Figuration eines Figuralen [...] eine herausgehobene Raum-ZeitFigur, die zwischen dem Einhalt und der Bewegung angesiedelt ist. Und eben in dieser Zwischensituation markiert die Pose jene Stelle, in der die Zeitlichkeit des Bildes sich einträgt. «19

Wie bereits beschrieben, hatte die Pose ihre historische Blütezeit im 18. und 19. Jahrhundert in den Tableaux vivants und Künsten der Attitüde. Anfang des 20. Jahrhunderts widmete sich der Kulturhistoriker Aby Warburg unter dem Begriff der Pathosformel den Gebärden und Posen der Bildwerke der Renaissance, welche auch als Vorbilder für ebenjene lebenden Bilder dienten. Die formelhaften Gebärden, Gesten und Posen antiker Bildwerke untersuchte er mit Blick auf ihr affektives Potenzial und ihre universelle Gültigkeit. ${ }^{20}$ In ihnen wird die historische Dimension der Bewegung im >Nachleben sichtbar: Die Vergangenheit bahnt sich über die körperliche Figuration der Affekte und Emotionen, über die stete Wiederkehr und Verwandlung in zeitlose Posen und Gesten, ihren Weg in die Gegenwart und Zukunft. Auf die Frage »nach den körperlichen Formen der nachlebenden Zeit [...] antwortet das bei Warburg absolut zentrale Konzept der Pathosformeln.«21

Innerhalb der Tanzwissenschaft bezeichnet die Pose das »Anhalten der Bewegung «, eine bewusst eingenommene »Pause« (von lateinisch pausa). ${ }^{22}$ Sie berührt daher verschiedene Stadien und Dimensionen der Zeitlichkeit: Neben der motorischen Bewegung des Tänzers / der Tänzerin, welche/r in einer gehaltenen Stellung gefangen ist und sich der veränderten Statik und Dynamik seines/ihres Körpers im Verharren in einer Position ausgesetzt sieht, ist auf die Rolle der Zuschauer/innen zu verweisen, deren Zeiterlebnis durch »Bewegungsphrasen, Tanzfiguren und komplexe Tänzerinteraktionen $\ll^{23}$

17 Vgl. ebd.

18 Gabriele Brandstetter, »Pose - Posa - Posing. Zwischen Bild und Bewegung«, in: dies., Brandl-Risi, Diekmann, Hold it!, S. 40-51, S. 45.

19 Ebd., S. 46.

20 Den Begriff der Pathosformel verwendet Warburg erstmals 1905 in dem Vortrag »Dürer und die italienische Antike«, abgedruckt in: ders., Werke in einem Band, hg. v. Martin Treml, Sigrid Weigel, Perdita Ladwig, Frankfurt am Main 2010, S. 176-183, S. 177.

Georges Didi-Huberman, Das Nachleben der Bilder. Kunstgeschichte und Phantomzeit nach Aby Warburg, Frankfurt am Main 2010, S. 212 f.

22 Brandstetter, Brandl-Risi, Diekmann, »Posing Problems«, S. 8.

23 Ebd., S. 9 . 
beeinflusst wird. Ihre Wahrnehmung eines Stücks und ihre Erinnerung daran sind diesen zeitlichen Gliederungselementen unterworfen. Zugleich kann jedoch das Erlebnis der Aufführung die Sukzession getanzter Bewegungen zu einer gefühlten Gleichzeitigkeit verdichten, wie Wilhelm Heinse bereits 1787 beschrieben hat. ${ }^{24}$

Als theoretische Figur hat die Pose insbesondere in der Fototheorie und der feministischen Lesart der Psychoanalyse Bedeutung erlangt. Zentrale Bezugspunkte sind dabei die Beschreibungen der Pose in der Fotogeschichte durch Roland Barthes, die von Craig Owens und anderen aufgegriffen wurde, und der Begriff des Blickregimes, den Kaja Silverman von Lacan entlehnt und der die Bedingungen des Gesehenwerdens im Rahmen kultureller, gesellschaftlicher und historischer Machtbeziehungen umfasst. Silverman beschreibt als Blickregime, ${ }^{25}$ wie dem Subjekt die Pose als $>$ In-Stellung-Gehen $<$ vor dem beobachtenden Blick aufgezwungen wird, hier dem $>$ Blick $<$ der fotografierenden Kamera. Dieses Posieren sei im Gegensatz zur allgemeinen Auffassung keine aktive Entscheidung, vielmehr sei der Handlungsspielraum des Subjekts eingeschränkt: »Das Subjekt >handelt< auf Geheiß der Kamera bzw. des Blickregimes; es reagiert auf die Unmöglichkeit, sein/ihr Gespiegeltwerden zu umgehen. ${ }^{26}{ }^{26}$ Fotografie ist Silverman zufolge eine viel zutreffendere Metapher für die Pose als beispielsweise die Filmkamera, ${ }^{27}$ denn die Fotografie ist für sie ebenso wie das Blickregime durch Bewegungslosigkeit und Tod gekennzeichnet, wie sie mit einem Zitat von Lacan deutlich macht: »Das Blickregime bricht nicht bloß die Bewegung ab, sondern läßt sie erstarren [...]. Seine Wirkung erweist sich darin, daß es die Bewegung einfängt und - tatsächlich Leben tötet. In dem Moment, wo das Subjekt in seiner Geste innehält, wird es mortifiziert. ${ }^{28}$ Craig Owens hat das Verhältnis der Kamera

24 Ebd. Die Autorinnen beziehen sich auf Heinses Ardinghello und die glückseligen Inseln, 1. Bd., 3. Teil, und datieren den Text fälschlicherweise auf 1780, er wurde jedoch erst 1787 veröffentlich. Vgl. die Originalausgabe Wilhelm Heinse, Ardinghello und die glückseeligen Inseln, Bd. 1, Lemgo 1787.

25 Kaja Silverman, »Dem Blickregime begegnen«, in: Christian Kravagna (Hg.), Privileg Blick. Kritik der visuellen Kultur, Berlin 1997, S. 41-64. Im englischen Original ist von »the gaze« die Rede (The Threshold of the Visible World, New York / London 1996).

26 Ebd., S. 49.

27 Deren Macht über die Darstellung des weiblichen Körpers im narrativen Film mittels kinematografischer Codes hat Laura Mulvey für die feministische Filmtheorie wegweisend dargelegt, vgl. Laura Mulvey, »Visual Pleasure and Narrative Cinema«, in: Screen 16/3, Herbst 1975, S. 6-18.

28 Jacques Lacan, Die vier Grundbegriffe der Psychoanalyse. Das Seminar von Jacques Lacan, Bd. Xl (1964), Olten / Freiburg im Breisgau, 1978, S. 125, zit. n. Silverman, »Dem Blickregime begegnen «, S. 44 . 
zum erstarrenden Körper als zeitliches definiert: Das Foto ist also die »Aufzeichnung eines früheren Innehaltens [...]. Ich erstarre [...], als würde ich das Standbild, zu dem ich gerade werde, vorwegnehmen; seine Opazität, seine Bewegungslosigkeit nachahmen; die Mortifikation des Fleisches durch die Fotografie in die ganze Körperoberfläche einschreiben.« 29

Die Wiederholung ist ein wesentliches Kennzeichen der Pose, da diese in ihrer stilisierten Organisation des Körpers und dem Einfrieren einer Bewegung sals Bild immer schon auf bekannte und im kollektiven Bilderrepertoire gespeicherte Bilder zurückgreift. Wenn jedoch der Blick der Kamera immer schon in der Pose vorweggenommen wird und beide, das beobachtende Subjekt und das beobachtete Objekt, sich der Vorgängigkeit des entstehenden Bildes bewusst sind, ist das Posieren ein Zustand, der eine Frage nach Original und Kopie unbeantwortet lässt und zugleich die zeitlichen Komponente der Wiederholung - das Wiederholen als eine Verbindung der Gegenwart mit der Vergangenheit - in der Frage nach Vorbild und Nachbild thematisiert: »Der Körper wird wahrnehmbar als immer schon Posierender, das Sehen als immer schon in Klischees Organisiertes. ${ }^{30}$ Die Zitathaftigkeit der affektiven Posen in Colemans So Different ... and Yet ist denn auch als Wiederholung von Zeichen im Sinne Derridas zu verstehen, als stereotype, iterable Gesten, deren Wirksamkeit auf keinen benennbaren Ursprung mehr zurückzuführen ist. ${ }^{31}$

Die Favorisierung der Fotografie als der Pose verwandtes Medium liegt nahe: Im unwillkürlichen Innehalten und Erstarren für den Moment der fotografischen Aufnahme nimmt das Subjekt sein Gesehenwerden vorweg, wird zum Posen imitierenden, fotografierten Objekt. Roland Barthes geht so weit, die »Natur der PHOTOGRAPHIE «32 in der Pose begründet zu sehen; sie ist eines der sechs Konnotationsverfahren, welche er als Codierungen der

29 Craig Owens, »Posieren«, in: Herta Wolf (Hg.), Diskurse der Fotografie. Fotokritik am Ende des fotografischen Zeitalters, Frankfurt am Main 2003, S. 92-114, S. 107 f.

$30 \quad$ Brandstetter, Brandl-Risi, Diekmann, »Posing Problems«, S. 13 .

31 Vgl. Jacques Derrida, »Signatur, Ereignis, Kontext«, in: ders., Randgänge der Philosophie, hg. v. Peter Engelmann, Wien 1988, S. 291-314.

32 Barthes, Die helle Kammer, S. 88. Hier gilt es zu beachten, dass für Barthes die Pose weder eine $»$ Haltung des photographierten Objektes noch eine Technik des operator [ist], sondern der Begriff für eine >Absicht« bei der Lektüre«. Als Betrachter sieht er in dem dargestellten Augenblick etwas Unbewegtes und projiziert diese Unbewegtheit auf »die in der Vergangenheit gemachte Aufnahme, und dieses Innehalten bildet die Pose « unabhängig davon, ob tatsächlich für das Bild posiert wurde. Hier konstatiert Barthes einen ersten Unterschied zwischen Fotografie und Film, denn seiner Ansicht nach wird die Pose »von der ununterbrochenen Folge der Bilder beseitigt und geleugnet« (vgl. ebd.). 
Fotografie, als deren zu dechiffrierende Botschaften, benennt. ${ }^{33}$ Silverman erklärt im Vergleich zum bewegten Bild: »The still photograph dramatizes more strikingly than the moving image both the advent of specular existence as the loss of flux and vitality, and that necessarily suspended state in which any body aspires to formal coherence. «34 Dennoch wäre es falsch, die Affinität der Fotografie zur Pose als Ineinssetzung zu verstehen, denn die Pose ist immer ein Zwischenzustand zwischen Bewegung und Erstarrung, sie ist »niemals eine statische Darbietung, das Foto, einmal aufgezeichnet, schon «. ${ }^{35}$ Ist vom Blickpunkt der finalen Stillstellung her die Ähnlichkeit und Verbindung von Fotografie und Pose zwar nicht von der Hand zu weisen, soll hier jedoch das Argument unterstützt werden, dass die Pose auch, vielleicht sogar gerade im bewegten Bild am schärfsten zutage tritt, denn in Film oder Video zeigen sich alle Stadien ihrer Figuration: der Beginn in einer Bewegung, ihre Entwicklung hin zur Pose, das Stoppen und Verharren, das unmerkliche Zittern der Glieder, an welchen Belastung und Anstrengung ebenso abzulesen sind wie Entspannung; schließlich das Lösen einer Stellung, die Beendigung des Innehaltens und Neuausrichtung des Körpers. Bettina Brandl-Risi beschreibt diese Besonderheit der Pose folgerichtig als »Bewegung in Latenz $«^{36}$ und verknüpft die »Unschärfe« im Zittern mit dem für die Entstehung und Wahrnehmung des Bildes notwendigen Blick der Zuschauenden, die in ständiger minimaler Bewegung befindlich sein müssen, um sehen zu können.

\section{Figuren und ihre Posen beiJames Coleman}

In So Different ... and Yet wird die Pose als körperliches und visuelles Pendant der weiblichen Rollen innerhalb der verschachtelten Erzählung der Darstellerin eingesetzt; ganz anderes die fotografischen Diaarbeiten, in denen die besondere Affinität der Fotografie zur Pose und das zugleich statische und bewegte Medium der Diaprojektion zusammentreffen.

Die Werke Seeing for Oneself (1987-1988), Charon (1989), Background (1991-1994), Lapsus Exposure (1992-1994), INITIALS (1993-1994) (Abb. 7) und Photograph (1998-1999) weisen einige Gemeinsamkeiten auf: Es handelt sich durchgängig um Diaprojektionen, bei denen mehrere Projektoren im Wechsel durch Auf- und Abblende einen sanften Übergang zwischen den einzelnen Bildern ermöglichen, zudem werden sie durch ein Voiceover begleitet. In

Roland Barthes, »Die Fotografie als Botschaft« (1961), in: ders., Der entgegenkommende und der stumpfe Sinn, S. 11-27, besonders S. 16-18.

34 Silverman, The Threshold of the Visible World, S. 198.

35 Brandstetter, Brandl-Risi, Diekmann, »Posing Problems«, S. 17.

36 Bettina Brandl-Risi, »Das Leben des Bildes und die Dauer der Pose«, in: dies., Brandstetter, Diekmann, Hold it!, S. 53-67, S. 62. 


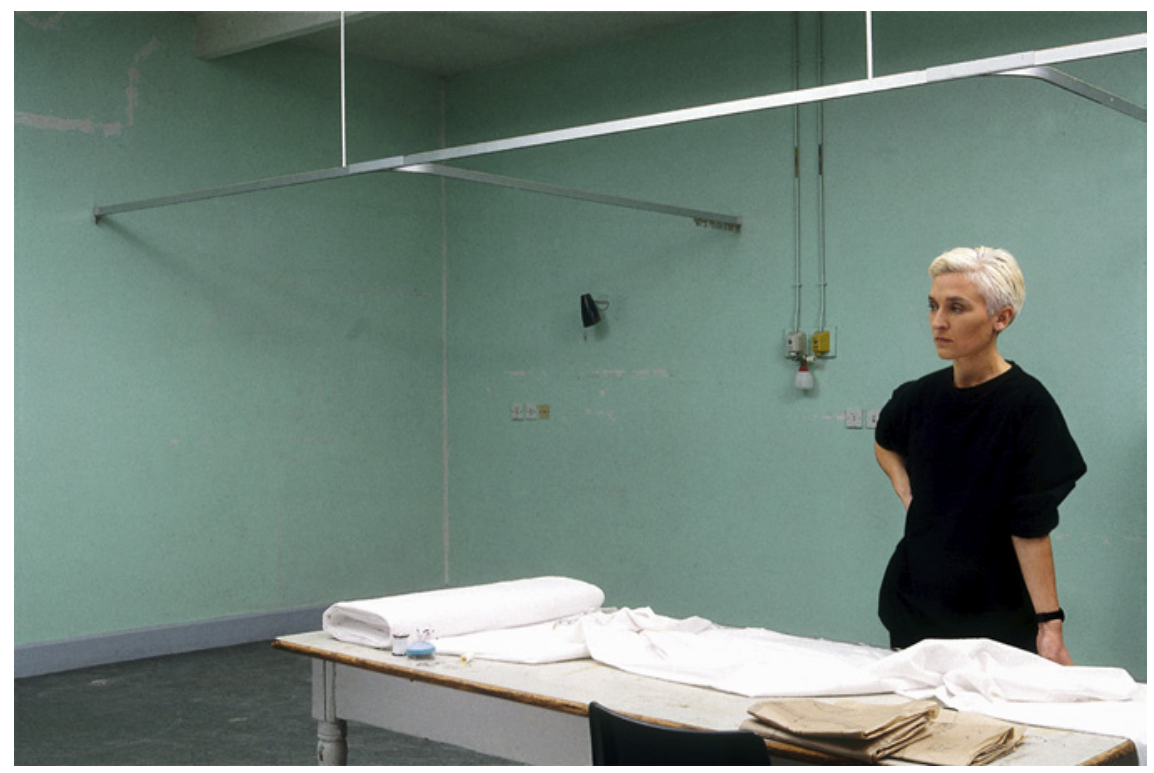

Abb. 7 James Coleman, INITIALS, 1993-1994 ( James Coleman) 
jeder Arbeit werden, wie in einer medialen Archäologie, Aspekte der Fototheorie, -geschichte und -ästhetik aufgegriffen und in einen mehr oder weniger narrativen Zusammenhang gestellt. Seeing for Oneself beispielsweise verknüpft die Erzählweise und Ästhetik des Fotoromans mit der englischen Gothic Novel, Charon besteht aus dreizehn Parabeln über die Paradoxien, die der Produktion und Rezeption von Fotografie innewohnen. ${ }^{37}$ Schließlich arbeitet Coleman stets mit Schauspieler/innen, die die Szenen in statischen Posen bevölkern. Hier sind deutliche Anleihen beim Tableau vivant zu beobachten. Alle genannten Werke sind bereits umfassend in der Forschung betrachtet worden: Analysen einzelner Aspekte finden sich unter anderem bei Jean Fisher, Dot Tuer, Benjamin H. D. Buchloh, Kaja Silverman und George Baker. ${ }^{38}$ Die deutschsprachige Monografie zu Coleman von Linda Schädler bietet detaillierte Beschreibungen und ebenfalls eigene Interpretationsansätze. ${ }^{39}$ Aus diesem Grund wird hier keine umfassende Beschreibung, Analyse und Interpretation jedes einzelnen Werks vorgenommen. Vielmehr wird auf die einzelnen genannten Aspekte fokussiert, die Colemans Werke dieser Periode verbinden, und gegebenenfalls auf die vorhandene Literatur verwiesen.

Seeing for Oneself (1987-1988) ist eine Mischung aus romantischer Novelle, Märchen, Familiendrama und Schauerroman. Erzählt wird die Geschichte von einer Sprecherin, deren rauchige Stimme den Eindruck eines Hörspiels hervorruft. Das erste Bild des >Châteaus<, eines Hotelbaus aus dem 19. Jahrhundert inmitten hoher Berge, ist in Farbe, die langsam an Sättigung verliert, so dass die Fotografie schwarz-weiß wird wie alle folgenden Bilder auch. Dies erweckt den Eindruck, dass eine Geschichte aus einer vergangenen Zeit erzählt wird: Eine junge Frau, Tamara, erreicht per Kutsche das Anwesen, in dem ihr Vater Neville, ein verschrobener Professor, mit ihrer missgünstigen Stiefmutter Clarice lebt. Ihr Vater hat im Keller des Hauses ein geheimes Labor eingerichtet, in dem er an einem Elixier arbeitet, das bei Verabreichung einen todesähnlichen Zustand hervorruft. Clarice hat ein Verhältnis mit J. B., einem jungen Mann, der zugleich Tamara Avancen macht. Neville wird von Clarice mit seinem eigenen Elixier getötet, jedoch wird das erhoffte Erbe Tamara

37 Vgl. Dot Tuer, »Blindness and Insight. The Act of Interrogating Vision in the Work of James Coleman «, in: Dia Center for the Arts (Hg.), Robert Lehman lectures on contemporary art, Nr. 1, New York 1996, S. 175-199, S. 179.

38 Jean Fisher, »Zu den neueren Arbeiten von James Coleman«, in: Dia Center for the Arts, James Coleman. Projected Images, S. 33-46; Tuer, »Blindness and Insight«; Buchloh, »James Coleman«; Kaja Silverman, »Melancholia 2«, in: Helmut Friedel (Hg.), James Coleman, Ausstellungskatalog Städtische Galerie im Lenbachhaus / Kunstbau München, München 2002, Ostfildern-Ruit 2002, S. 16-32; Baker, »Reanimations (I)«.

Schädler, James Coleman und die Anamorphose. 
übertragen, die den Tod ihres Vaters betrauert. Sie zweifelt an seinem natürlichen Tod und äußert dies gegenüber einem jungen Arzt namens Murray. Der Leichnam ist aus unerklärlichen Gründen verschwunden. Auch Tamara wird bald von Clarice vergiftet, überlebt den Anschlag jedoch und erwacht wieder. Murray begleitet sie in das unterirdische Labor, wo sie Aufzeichnungen ihres Vaters an sich nimmt. Im Schloss begegnet sie Clarice, die glaubt, einen Geist zu sehen und sie mit einer alten Lanze erstechen will. Sie trifft jedoch stattdessen J. B., der stirbt. Das Personal der Geschichte tritt in historischen Kostümen auf, die Szenen spielen sich in repräsentativen Räumen vor dunkel getäfelten Wänden, im Speisesaal oder vor grobem Mauerwerk ab. Die Arrangements der Figuren gleichen Darstellungen aus Interieurs oder Konversationstücken des 18. Jahrhunderts, in denen die Beziehungen und Hierarchien zwischen Personen mittels Posen und Gesten deutlich gemacht werden. Die Haushälterin Polly dient als Erzählerin und Identifikationsfigur für den Betrachter / die Betrachterin, indem sie verschiedene Szenen heimlich beobachtet und kommentiert. Es scheint kein Zufall zu sein, dass in der erzählten Geschichte alles um das Thema Lebendigkeit kreist, die auch für das Tableau vivant, das lebende Bild, elementar ist. Die spezielle Wirkung der Tableaux vivants beruhte auf der Schwierigkeit, statische Szenen mit lebendigen Darstellern nachzustellen, deren Atmen und Zittern den entstehenden Bildern das Leben einhauchte und ihren Reiz ausmachte. Eine Schlüsselszene von Seeing for Oneself findet ein anschauliches Bild dafür: Tamara wird durch das Elixier ihres Vaters in einen todesähnlichen Zustand versetzt und von Polly, welche sie findet, für tot gehalten. Tamara ist zwar bei Bewusstsein, kann sich aber nicht bewegen, wie die Sprecherin mit gehauchter Stimme suggeriert: »I can't feel my body, I can't move! [...] My eyes are open, but I don't see, I cannot speak.« Mittels eines Spiegels, an dem der Atem kondensieren soll, versucht der Arzt Murray herauszufinden, ob Tamara lebt; er hegt große Zweifel an ihrem Tod und will den Körper genauer untersuchen. ${ }^{40}$ Schließlich erwacht Tamara jedoch wieder.

40 Das Sehen mit den eigenen Augen - seeing for oneself -, die Überprüfung der Wahrheit durch medizinische und wissenschaftliche Methoden in der klinischen Pathologie erfolgte früher oft in großen Hörsälen, den sogenannten anatomischen Theatern. Jean Fisher zieht hier eine Parallele zum populären Theater, in dem ebenfalls der Körper als Spektakel zur Schau gestellt wird. Sie betont in ihrer Analyse des Werks die Rolle der Schlossarchitektur, die von Neville, dem Alchemisten, Architekten und Philosophen, nach dem Vorbild der menschlichen Anatomie entworfen wurde. Das Herzstück dieses Organismus sei sein Labor, in dem die Formel, die über Leben und Tod entscheiden soll, entwickelt wurde. Vgl. Jean Fisher, »On Seeing for Oneself. A Perspective«, in: Michael Asher / James Coleman, Ausstellungskatalog Artists Space, New York 1988, S. 20-26, S. 21-23. Dieser Gedanke wird aufgegriffen von Frédéric Migayrou, »Le cas de figures«, in: 
Die Personen in Seeing for Oneself erinnern in ihren historischen Kostümen und steifen Posen an typische Bilder aus der Frühzeit der Fotografie, in der minutenlanges Posieren aufgrund der langen Belichtungszeiten notwendig war. In anderen Arbeiten greift Coleman auf weitere Stereotypen der fotografischen Pose zurück: das Selbstporträt und Porträtaufnahmen im familiären Kreis (Charon), den Tanz (Photograph) und das Theater (INITIALS). Dass Colemans Figuren stets eine Versunkenheit und körperliche Stasis ausstrahlen, die angesichts heutiger Möglichkeiten der Fotografie technisch nicht notwendig sind, entlarvt diese als bildliche Stilmittel. Fotografische Stasis, wie sie Coleman bewusst einsetzt, hat für ihn offensichtlich die Funktion, das Verhältnis zwischen Körper, Bild, Figur und Narration neu zu definieren, wie auch George Baker vermutet: »Innerhalb der Bilder werden die Posen von der erzählerischen Motivation befreit, werden zu einer Reihe sich entwickelnder >Verhaltensweisen<, die eine nahezu autonome und unverfälschte >Theatralisierung der Körper< hervorbringt, unabhängig von einer jeden vorgegebenen Rolle. « ${ }^{41}$ Die Theatralisierung der Körper - diesen Ausdruck entlehnt Baker bei Gilles Deleuze - bedeutet, wie Baker an seinem Beispiel INITIALS beschreibt, eine Loslösung der figurativen Darstellung von ihrer narrativen Verankerung und eine Besinnung auf visuelle Merkmale:

In solchen Momenten fangen die üblicherweise voneinander geschiedenen Figuren der Szenen an, visuelle Merkmale miteinander zu `teilen<, wenn sie auch nie den Blick des anderen treffen; sie >reimen $<$, stellen einen Gleichklang her zwischen Armgesten oder -posen, berühren dasselbe Objekt oder sehen es an, oder sie reihen sich signifikanterweise entlang von Schräglinien auf. ${ }^{42}$

Zwar weisen solche bildlichen Elemente, das heißt Blickachsen von Figuren oder die Parallelisierung von Bewegungen, auf die malerische Tradition zurück, doch diese will Baker für Coleman nicht gelten lassen. Anders als beim Gemälde kann der Betrachter / die Betrachterin bei Coleman nämlich keinen zentralen Standpunkt vor dem Bild einnehmen, da dort der Projektor platziert ist und er oder sie das Bild verdecken würde. So gleichen sich nicht nur die schrägen Blicke der Darsteller/innen aus dem Bild heraus, die Rosalind E. Krauss als double face-out beschrieben hat, sondern sie werden in der seitlichen Sicht des Zuschauers / der Zuschauerin gespiegelt. Das double face-out ist für Krauss eines der wesentlichen Merkmale von Colemans

James Coleman, Ausstellungskatalog Musee d'Art Moderne de la Ville de Paris, Paris 1989,

S. 7-37, S. 27.

41 Baker, »Reanimations (I)«, S. 64.

42 Ebd., S. 61. 
Diaarbeiten, und sie beschreibt diese Konstellation vor allem am Beispiel von Seeing for Oneself und INITIALS. ${ }^{43}$ Wie in den klassischen Fotoromanen oder Comics, welche ökonomisch mit dem in einem Buch oder einer Zeitschrift zur Verfügung stehenden Platz haushalten mussten, werden hier Aktion und Reaktion zweier oder mehrerer Personen in einem Bild zusammengefasst. Was im Film durch eine Schuss-Gegenschuss-Montage gelöst werden würde, wird in diesem Medium, welches die bewegliche Projektion des Films mit der Stasis der Fotografie verbindet, zum eigenen syntaktischen Element, denn der Dialog zweier Personen wird durch das Aneinander-Vorbeigehen der Blicke in eine einzelne Aufnahme übersetzt. Alle Blicke gehen somit ins Leere aus dem Bild heraus, doch sie sprechen dabei die auch axial verschobenen Zuschauer/ innen direkt an. Wie Baker richtig an Krauss kritisiert, wird hier nicht nur eine bildliche Verflachung der Szene durch die Unterminierung der suture erreicht, sondern zugleich auch eine Öffnung, da die Blicke, die aus dem Bild herausführen, den Betrachter / die Betrachterin wieder ins Geschehen hineinholen. ${ }^{44}$ Die Posen erreichen tatsächlich eine zugleich narrative und nichtnarrative Wirkung: Sie schließen die Betrachter/innen aus Szenen aus, in denen die Kommunikation durch die aneinander vorbeiführenden Blicke zum Stillstand kommt; gleichzeitig transportieren aber die theatralen Gesten, minimale Richtungsandeutungen und Parallelen in der Haltung oder Ähnlichkeiten in der Kostümierung eine eigene, bildliche Erzählung. Vergleichbar ist dies mit dem Stillstand im Film, wie ihn Giorgio Agamben beschreibt: In ihm liege der Unterschied zwischen Film und Erzählung begründet, »der narrativen Prosa, mit der man den Film gerne vergleicht. Die Stillstellung zeigt uns jedoch, dass der Film der Poesie näher steht als der Prosa.« ${ }^{45}$ Wie eine lautliche, metrische Grenze innerhalb der Poesie schaffe Stillstand im Film für kurze Zeit eine Zäsur, die »das Bild selbst bearbeitet, es der Herrschaft des narrativen Vermögens entzieht, um es als solches vor Augen zu stellen« ${ }^{46}$

Coleman nimmt, so wird deutlich, den Körper und sein Eingebundensein in Narrative, Blickregimes und Architekturen als Ausgangspunkt für seine

43 Krauss, » ... And Then Turn Away?««, S. 21-23, und dies., »Reinventing the Medium«.

44 Vgl. Baker, »Reanimations (I)«, S. 58-6o. Suture ist ein Begriff aus der psychoanalytischen Filmtheorie und meint die Einbindung der Filmzuschauer/innen in die filmische Fiktion über die Identifikation mit dem filmischen Subjekt, welche durch Schuss-GegenschussFormationen und die Narration erreicht wird. Vgl. u. a. Jean-Pierre Oudart, »Cinema and Suture«, in: Screen 18/4, Winter 1977/78, S. 35-47; Stephen Heath, »Notes on Suture«, in: Screen 18/4, Winter 1977/78, S. 48-76.

45 Giorgio Agamben, »Wiederholung und Stillstellung«, in: Françoise Joly (Hg.), Documenta X. documents 2, Ostfildern-Ruit 1997, S. 72-75, S. 74.

Ebd. 
Tableaus, in deren Ästhetik die statischen Posen, das theaterähnliche Arrangement der Darsteller/innen und ihre besondere Blickführung das Vokabular bilden. Coleman knüpft damit an die vorwiegend in feministischen Kreisen geführte Diskussion um die Unterwerfung des weiblichen Körpers unter das Regime des männlichen Blicks an, jedoch völlig anders als die radikale Performance und Body-Art von Künstler/innen wie Carolee Schneemann, VALIE EXPORT, Marina Abramović, Pipilotti Rist, Vito Acconci, Chris Burden und Victor Bruns: Er versucht nicht, die Politisierung des Körpers durch Eingriffe in diesen, durch Verletzung oder Geißelung zu offenbaren, sondern wendet sich den systemischen Regimes der Unterwerfung zu. Jean Fisher formuliert dies wie folgt:

Like many artists whose work evolved from the experiences of the late '6os, Coleman understood that the site of conflict, the place where the multiple and paradoxical sociopolitical narratives intersected, was the body itself. While other artists addressed the issue directly through performance art, Coleman worked at undermining the politics of representation as it organized space, the body, and the individual's perception of the world according to prescribed parameters. ${ }^{47}$

Coleman findet mit den stummen Posen seiner Darsteller und Darstellerinnen ein anschauliches Bild der Verstrickung des Körpers in Geschichten und Geschichte, in die Narrative populärer Konsumkultur und die Historizität der Medien. Innerhalb seiner »Archäologie des Spektakulären« erzielt Coleman, wie Buchloh zusammenfasst,

eine Untersuchung der komplizierten Bezüge zwischen der Geschichtlichkeit des skopischen Verlangens und seiner mannigfachen historischen Ausbildungen (in der Malerei, im Theater, in der Fotografie, im Film) und der späteren Desublimierung und dem Entschwinden dieser Erfahrungsformen und deren unaufhaltbarer Zerstreuung und Zerstörung in den Praktiken der Massenkultur. ${ }^{48}$

An den Posen der Darsteller/innen, welche die fotografische Fixierung in ihrer Unbeweglichkeit bereits vorwegnehmen und die dann jedoch im dynamischen Medium der Diaprojektion erneut beweglich werden, treffen die unterschiedlichen zeitlichen Dimensionen zusammen: die Geschichtlichkeit der Narrativität und des historischen Kontextes, die Zeitlichkeit der Medien und die Zeitlichkeit des arretierten Körpers, der unter dem Blick der Kamera wie auch der Betrachtenden innehält.

47 Fisher, »On Seeing for Oneself«, S. 20-26.

48 Buchloh, »James Coleman«, S. 94. 


\section{Die Pose des Tanzes im Film. Tacita Dean, Merce Cunningham performs STILLNESS (2008)}

Tacita Deans Installation Merce Cunningham performs STILLNESS (in three movements) to John Cage's composition 4'33" with Trevor Carlson, New York City, 28 April 2007 (six performances; six films) von 2008 thematisiert die Konfrontation von Bewegung und Stillstellung im Bild auf eine außergewöhnliche Weise. Das bewegte Bild des Films trifft auf die Körperlichkeit des Performers in der gehaltenen Pose, und beide behandeln mit den ihnen eigenen Mitteln die Stillstellung; es offenbart sich eine konzeptuelle Einstellung zu Zeit und Darstellung in Film und Performance. In Merce Cunningham performs STILLNESS porträtiert Tacita Dean den Tänzer und Choreografen Merce Cunningham, welcher bis zu seinem Tod im Jahr 2009 noch aktiv mit seiner Kompanie arbeitete. Die sechsteilige Filmarbeit mit Leinwänden in verschiedener Größe zeigt den greisen Cunningham in seinem New Yorker Tanzstudio, von verschiedenen Blickpunkten aus und mit unterschiedlichen Einstellungen gefilmt. In der Veröffentlichung zur Ausstellung der Arbeit im Sprengel Museum Hannover 2010 berichtet Tacita Dean, dass sie Cunningham um die Erlaubnis gebeten hatte, einen Film über ihn zu drehen, und mit ihm übereingekommen war, dass er eine Performance zu dem sstillen Stück 4'33" (1952) von John Cage, dem Komponisten und langjährigen Lebens- und Arbeitspartner von Cunningham, aufführen würde. Sie wusste jedoch bis zum Beginn der Aufnahmen nicht, wie diese aussehen würde.

Cunningham machte sechs Aufführungen für die sechs Filme der Arbeit. Diese sechs Filme werden in der Rauminstallation gleichzeitig auf verschieden große durchscheinende Leinwände, die am Boden stehen, projiziert. Jeder Film zeigt einen anderen Ausschnitt aus dem Trainingsstudio der Company in New York, wobei im Bildmittelgrund stets Cunningham, in farbigem Hemd und Turnschuhen, auf einem Klappstuhl sitzt. Der Raum ist erfüllt von Straßengeräuschen, die von außen in das Studio dringen, sowie von den Geräuschen der Projektoren. Am Spiegel sieht man die Spuren und Abdrücke der Tänzer, das Studio ist deutlich ein Ort der körperlichen Arbeit. Cunningham sitzt regungslos auf seinem Stuhl und schaut geradeaus, je nach Blickwinkel und Position der Kamera in deren Richtung oder auch von ihr weg. Sein Blick fängt aber die Person von Trevor Carlson ein, dem Direktor seines Studios. Carlson blickt, wie man in manchen der Filme sehen kann, auf eine Stoppuhr und beginnt nach einer Weile, lautlos mit einer Geste seiner rechten Hand fünf Sekunden herunterzuzählen. Dies ist das Signal für Cunningham, seine Position leicht zu verändern, beispielsweise einen Arm anzuwinkeln oder den Kopf leicht zu drehen, das Gewicht zu verlagern. Nach weiteren Sekunden, an deren Ende 
Carlson erneut herunterzählt, verändert Cunningham seine Position abermals. Nach diesem dritten Durchgang und ca. 4 Minuten, 33 Sekunden, ist der Film beendet und wird dann, als Loop, erneut projiziert.

Die Installation führt alle sechs Filme parallel auf, aber nicht zeitlich synchron, das heißt die Bewegungen sind leicht versetzt, jedoch nicht nach einem erkennbaren Muster; sie werden auf verschieden große Leinwände projiziert, welche in einem großen Raum gegeneinander verschoben aufgestellt sind, jedoch immer im rechten Winkel zueinander (Abb. 8). Somit wird der Raum durch die Leinwände als transparente Wände und die davorstehenden Filmprojektoren architektonisch gegliedert. Dies ermöglicht dem Besucher oder der Besucherin auch, mehrere Filme zugleich zu sehen. Die Größe der Leinwände reicht von ca. $3 \times 4$ Metern bis zu ca. $80 \times 6$ o Zentimetern, einer einzigen Glasplatte, die auf Augenhöhe hängt und einen Porträtausschnitt von Cunninghams Kopf zeigt. Durch den Abstand der Kamera zum Tänzer und die verschieden große Projektion erscheint Cunningham in jedem Film lebensgroß.

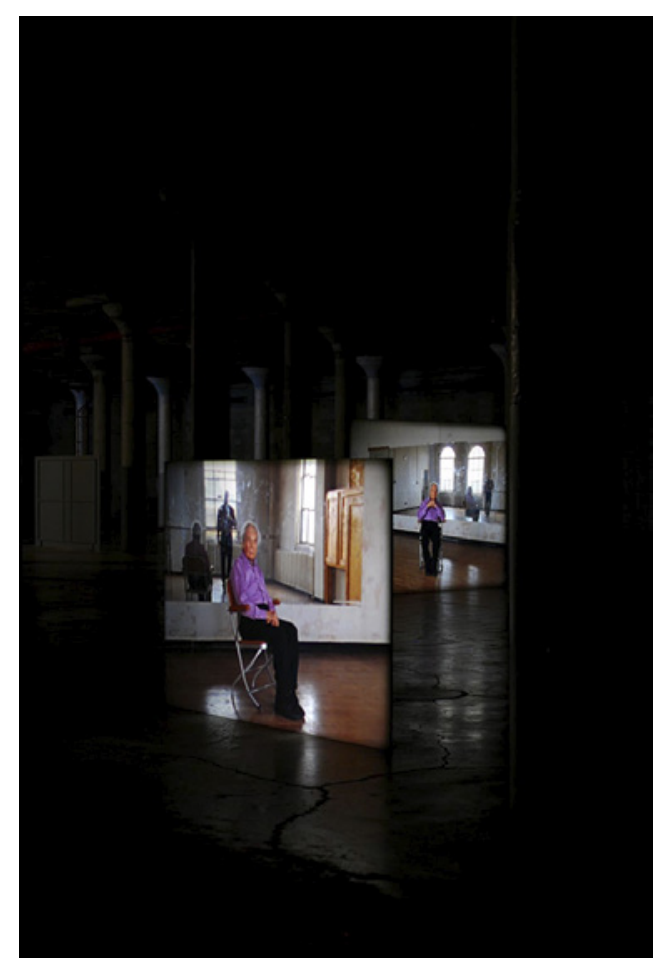

Abb. 8

Tacita Dean, Merce Cunningham performs STILLNESS ...

(six performances, six films), 2008, Ausstellungsansicht Dia:Beacon, New York, 2008 
Merce Cunningham war einer der wichtigsten Vertreter des Modern Dance seit den 195oer Jahren. In Abgrenzung zu Martha Graham, bei der er seine Ausbildung genoss und in deren Kompanie er tanzte, führte er mit seinen Solostücken und ab 1953 mit seiner eigenen Kompanie einen gänzlich neuen Stil und eine neue Arbeitsweise in den Modern Dance ein. Wie Trisha Brown und später William Forsythe stehen für Cunningham nicht Expressivität und Emotionalität des Tanzes im Vordergrund, sondern allein das Bewegungspotenzial des menschlichen Körpers: »Das Medium des Tanzes ist für Cunningham mit der Bewegung selbst identisch. ${ }^{49}$ In Cunninghams Arbeit gibt es, und das ist interessant für seine Darstellung durch Tacita Dean und vielleicht eine Parallele zwischen den beiden, keine ssymbolische< Ebene, welche auf die dahinterliegende Bedeutung der physischen Oberfläche der Körper verweisen würde - sie sind reine körperliche Bewegung. Seine Choreografien zeichnen sich durch die Aneinanderreihung abstrakter Bewegungsmuster aus, in denen nicht der Ausdruck, sondern die Bewegungsqualitäten wichtig sind: Es geht um das Gewicht des Körpers und seine Verteilung im Raum, um Tempo, Rhythmus und immer wieder um die Wiederholung von Bewegungsabläufen und kleinen Phrasen, die vom Solotänzer / von der Solotänzerin zur Gruppe und zurück wechseln. Wenn er mit Musik arbeitet, spielen sich die Aufführungen nie im Einklang mit der Musik, sondern immer nur parallel zu ihr ab. Dementsprechend trainieren die Tänzer/innen auch nicht zur Musik, sondern verinnerlichen Tempo und Rhythmus des jeweiligen Stückes durch das intensive Training mit der Stoppuhr. ${ }^{50}$ Die Stücke sind weder narrativ noch weisen sie eine nachvollziehbare Dramaturgie auf, weshalb sie auch abwertend als formalistisch bezeichnet wurden. ${ }^{51}$ Sabine Huschka beschreibt die Besonderheit von Cunninghams Arbeit mit der Bewegung wie folgt:

Anstatt dem Tanzkörper eine expressive Funktion einzuräumen, wird diese radikal ausgeschlagen. Cunninghams bewegungstechnische Prinzipien repräsentieren keine Grunderfahrungen der menschlichen Existenz, wie sieHumphrey suchte, oder trainieren den Körper bis zu jenem Grad von Beherrschung, an dem er ein Anderer wird, wie es Grahams theatraler Tanzkonzeption vorschwebt. Die Cunningham-Technik trainiert ein hoch artifizielles Bewegungsrepertoire, das den Körper von jeglichem weihevollen Dienst freispricht [...]. Konzentriert nimmt Cunningham indessen im kinetischen Möglichkeitsfeld des Körpers Zuflucht. ${ }^{52}$

\footnotetext{
49 Sabine Huschka, Merce Cunningham und der Moderne Tanz. Körperkonzepte, Choreographie und Tanzästhetik, Würzburg 2000, S. 14.

$5^{\circ} \quad$ Julia H. Schröder, Cage \& Cunningham Collaboration. In- und Interdependenz von Musik und Tanz, Hofheim 2011, S. 155.

$5^{1}$ Huschka, Merce Cunningham und der Moderne Tanz, S. 239.

52 Ebd.
} 
Gerade aufgrund seiner Loslösung von der Ausdrucksdimension des Modern Dance gilt Cunningham als einer der großen Erneuerer des Tanzes.

Cunninghams Performance für Tacita Deans Filminstallation ist eine Hommage an John Cage und dessen Arbeit mit Zeit und Geräuschen, welche die zeitgenössische Musik revolutionierte; Cunningham interpretiert in allen sechs Filmen das Stück 4'33" von Cage aus dem Jahr 1952. 4'33" wurde am 29. August 1952 in Woodstock, New York, von David Tudor uraufgeführt und von Cage selbst als sein bedeutendstes Stück bezeichnet. ${ }^{53}$ Die drei Sätze Stille (»Tacet«) werden durch das Schließen und Öffnen des Klavierdeckels angezeigt. Die in der Notation nicht angegebene Dauer der Sätze erwürfelte er vor der Aufführung und erhielt so 33 Sekunden, 2 Minuten und 40 Sekunden sowie 1 Minute und 20 Sekunden, was eine Gesamtlänge von 4 Minuten, 33 Sekunden ergibt. ${ }^{54}$ Wesentlich an dem Stück sind die zufälligen Geräusche, die während der Sätze durch die Umwelt und die Zuhörer selbst entstehen und laut Cage »eine Musik erzeugen, die weitaus interessanter ist als die Musik, die man im Konzertsaal hört «. ${ }^{55}$ Cunningham führte $4{ }^{\prime} 33^{\prime \prime}$ nicht das erste Mal auf: Bereits 1992, als John Cage starb, integrierte er drei statische Posen in sein Stück Enter, an dem er gerade arbeitete. Später choreografierte er zu Ehren von Cage eine Aufführung, die auf $4^{\prime} 33^{\prime \prime}$ basierte. ${ }^{56}$ In dieser hielten fünf Tänzer jeweils selbstgewählte Posen für die Zeit der drei Sätze des Stücks. Dennoch ist Merce Cunningham performs STILLNESS keine Wiederaufführung, sondern eine neue Arbeit, in der Cunningham in enger Zusammenarbeit mit Dean und ihrer Kamera seine Interpretation der Komposition entwickelt.

Die Ausstellungsarchitektur bewirkt, dass die Geräusche aus dem Off, die für Cage die Musik seiner Stücke darstellten, sich mit den Geräuschen der Projektoren und der Besucher/innen vermischen. Die Kadrierung der Filme gibt die Quellen der Geräusche nicht preis und lässt so den nicht sichtbaren Teil der gefilmten Welt in der Imagination der Betrachter/innen entstehen.

53 Richard Kostelanetz, John Cage im Gespräch. Zu Musik, Kunst und geistigen Fragen unserer Zeit, Köln 1989, S. 63 .

54 James Pritchett beschreibt, wie Cage die Idee zu dem Stück bereits Jahre vorher hatte und dass die Werke der Jahre zwischen 1948 und 1952 von der »idea of structured silence« geprägt waren: »Thus considered, $4^{\prime} 33^{\prime \prime}$ represents an instance of a truly empty rhythmic structure.« In der Revision des Stückes 4'33" von 196o falle der Fokus auf die Struktur indes weg. Vgl. James Pritchett, The Music of John Cage, Cambridge 1993, S. 59 f., hier S. 6o, und Anm. 24, S. 209. Ebd.

$5^{6}$ Julia H. Schröder, »So that one becomes aware of the presence of a sound - or it's absence «. Circling Cage's Concept of `Silence « «, in: Dieter Daniels, Inke Arns (Hg.), Sounds like silence:John Cage 4'33" silence today: 1912-1952-2012, Leipzig 2012, S. 59-66, S. 64. 
Ursula Frohne hat hervorgehoben, dass die filmische Kadrierung, die den Ausschnitt aus der vergangenen Realität zeigt, dabei aber immer auch auf das nicht Gezeigte verweist, eine Parallele in der Verschränkung von Filmraum und Ausstellungsraum hat: »Ebenso wie die Kadrierung im Film auf einen Raum im Off, auf das hors champ verweist, welches nicht sichtbar, aber gegenwärtig ist und als imaginärer Raum die Vorstellung des Zuschauers besetzt, entsteht ein Beziehungsgeflecht zwischen dem filmimmanenten Suggestionsraum und dem topologischen Installationsraum. ${ }^{57}$

Merce Cunningham performs STILLNESS ist eine Arbeit über Zeit und zugleich über Musik beziehungsweise die Abwesenheit von Musik und die Anwesenheit der Geräusche, die bei der Stille des Instruments hörbar werden. Aber gleichzeitig ist es eine Arbeit über Bewegung, die in Cunninghams oben erläuterter Konzeption des Modern Dance eine entscheidende Rolle spielt, und über deren Sichtbarkeit in der Körperlichkeit des Tänzers. Tacita Deans Filminstallation steht im Dialog mit beiden Aspekten und stellt ihnen die analoge Zeit des filmischen Mediums, seiner Aufzeichnung und Wiedergabe, und der Rezeptionszeit der Betrachter/innen in der Installation gegenüber. Die Bewegungslosigkeit des Tänzers, die nie statisch ist, sondern als gehaltene Pose die Dynamik der Bewegung nie ganz aufgibt, die Stille des Musikstückes voller Geräusche und die langsame Filmästhetik Tacita Deans mit ihren langen Einstellungen und dem analogen Bearbeitungsprozess korrespondieren und ergänzen einander. Wie die drei Konzeptionen von Zeit über die Bewegung und Stillstellung ineinandergreifen, soll im Folgenden untersucht werden - zunächst mittels der Analyse von Cunninghams Posen als angehaltene Bewegung im Vergleich zum >stillgestellten< Film, mit Blick auf Cunninghams Tanzästhetik und seinen Umgang mit Zeit sowie im Hinblick auf die theoretische Diskussion von Performance und Performativität des Film beziehungsweise in demselben.

\section{Stillstellung in Tacita Deans Filmen}

Die Wirkung von Merce Cunningham performs STILLNESS gründet zunächst nicht auf der körperlichen Präsenz von Cunningham - hierauf soll weiter unten genauer eingegangen werden -, sondern auf der materiellen Präsenz aller Bestandteile der Installation in Form von Leinwänden und Projektionsapparaten. Tacita Dean verwendet 16-mm-Film und die entsprechenden

57 Lilian Haberer, Ursula Frohne, »Kinematographische Räume. Zur filmischen Ästhetik in Kunstinstallationen und inszenierter Fotografie«, in: dies., Kinematographische Räume, S. $9-52$, S. 21. 
Projektoren, welche den Ausstellungsraum mit ihrem beständigen Rattern und Sirren erfüllen. Schon bevor die Aufmerksamkeit der Betrachter/innen auf die filmische Darstellung gerichtet wird, fallen die spezielle Ästhetik des analogen Films, sein Zittern, die besondere Körnigkeit mit ihrer leichten Unschärfe sowie die kräftigen Farben ins Auge. Dean hat wiederholt die Bedeutung der Zeitlichkeit des analogen Films für ihre Arbeit betont: Film als »Medium der Zeit «58 stellt für sie die materielle Entsprechung der Zeit der realen Dinge dar, deren Poesie nur im analogen Medium eingefangen werden kann, welches selbst >atmet und lebendig ist, seine eigene Zeitlichkeit hat. Die Zeitlichkeit des Films beinhaltet ihrer Auffassung nach die Zeit der Aufnahme, die bereits durch die Spulenlänge begrenzt ist, die Zeit der physikalischen Bearbeitung des Materials durch Schnitt und Montage, die Zeit der Betrachtung sowie die individuelle Wahrnehmung des Leinwandgeschehens. Ihr Stilmittel der langen, statischen Einstellungen erzeugt eine subjektive Verlangsamung des Films, die den Betrachter oder die Betrachterin auf seine/ihre eigene Wahrnehmung von Zeit aufmerksam werden lässt. ${ }^{59}$ Deans Filme werden daher häufig unter dem Stichwort der >Stillstellung des Films $<$ besprochen; gemeint ist hier die Wirkung der statischen Einstellungen ohne filmische Mittel wie Schwenk und Zoom, mit wenigen Schnitten und meist ohne erkennbare Handlung. Dass sie Geschehen aufnimmt, das sich vor der Kamera ereignet, und dieses in Realzeit wiedergibt, wiederspricht den Sehgewohnheiten zeitgenössischer Betrachter/ innen und erscheint somit als langsam oder stillgestellt. Dabei zeigen Deans Filme durchaus Bewegung; handeln sie jedoch beispielsweise von Naturphänomenen wie der Bewegung des Wassers oder des Lichts, werden diese so langsam dargestellt, dass die kurze Verweildauer durchschnittlicher Ausstellungsbesucher/innen nicht ausreicht, um die Veränderungen wahrzunehmen. Filme, in denen Personen vorkommen, zeigen diese bei alltäglichen Handlungen, die aber unkommentiert für sich stehen und in ihrer Bedeutung für die Person oder für Tacita Dean erst in den die Arbeit begleitenden Texten offensichtlich werden.

Durch die unbewegten Kameraeinstellungen, die fehlende Intentionalität der Aktionen und die Präsentation der Filme in Ausstellungen (und nicht im Kino) unterscheidet sich die Art der Betrachtung der Filme von der eines snormalen< Filmpublikums: Die Aufmerksamkeit wird von kleinen Details

$5^{8}$ Tacita Dean in Rina Carvajal, »Film is a Medium of Time. A Conversation with Tacita Dean«, in: Briony Fer (Hg.), Tacita Dean. Film Works, Ausstellungskatalog Miami Art Central, Miami 2007, Mailand 2007, S. 45-63, S. 48.

59 Vgl. ebd. S. 57 f., sowie Tacita Dean, »Kodak. Analogue«, in: Fer, Tacita Dean, S. 96-97, S. 96 . 
gefesselt, das Auge hat Gelegenheit zu schweifen, das Verhältnis von Formen zueinander und Texturen der Flächen wahrzunehmen. Die visuelle Wahrnehmung ähnelt somit stärker der eines Gemäldes oder einer Fotografie. Achim Hochdörfer hat den Einfluss von Schnitt und Länge der Einstellungen auf die Zeitlichkeit der Wahrnehmung bei Tacita Dean treffend beschrieben:

Obwohl Tacita Deans Filme keine Montagen im klassischen Sinne sind, beharren sie gleichwohl auf ihrer Montiertheit. Man könnte von einer >Losigkeit< der einzelnen Bildsequenzen sprechen, die sich immer wieder von dem narrativen Zusammenhalt lösen und ein Eigenleben beanspruchen. Diese >Losigkeit< resultiert nicht zuletzt daraus, dass sie meist zwischen zehn und 40 Sekunden dauern - gerade so lange also, dass sie als Bild gelesen werden und die Orientierung im narrativen Verlauf verloren geht. Es kann auf diese Weise kein Rhythmus aufkommen, der den nächsten Schnitt bereits gedanklich vorwegnimmt (was bei einem kürzeren Abstand der Fall wäre). Das Auge lässt sich auf jede Bildeinstellung ein, auf ihre Komposition, ihre minimalen Bewegungen und auf ihre filmische Materialität; und erst in dem Moment, in dem der zeitliche Horizont vage wird und sich ein Gefühl für >Zeitlosigkeit< eingestellt hat, erfolgt der nächste Schnitt. [...] Seine Plötzlichkeit erweist sich deshalb immer auch als eine vergangene, als verlorener Augenblick, als nachträgliche Präsenz. ${ }^{60}$

Die Wahl der Bildausschnitte und die Komposition von Gegenständen weisen deutliche Bezüge zur Malerei auf. Deans vielfältige zeichnerische Arbeiten, die sie vorwiegend mit Kreide auf schwarzen oder grünen Tafeln anfertigt, zeugen davon, dass sie ihre Bilder nicht aus einer Logik des Filmischen, auf dem Prinzip der Montage basierend, entwickelt, sondern dass sie als bildende Künstlerin von der Flächigkeit und räumlichen Organisation des Bildfeldes her denkt. Stillstellung in diesem Sinne bedeutet also nicht das Anhalten von Bewegung, sondern die Reduktion dessen, was Deleuze >sensomotorische Bewegung < genannt hat - die sichtbare Bewegung, die auf einem AktionsReaktions-Schema beruht und innerhalb eines Films in Handlungszusammenhänge eingebettet ist. ${ }^{61}$

Aus den bisherigen Ausführungen zur Rolle der Zeit in Tacita Deans Filmen lässt sich zugleich ein materialbasiertes und ästhetisches Verständnis von Filmzeit ableiten. Dean knüpft dabei an eine lange Tradition des Experimentalfilms an, die an dieser Stelle nur kurz skizziert werden soll, da sie von der eigentlichen Diskussion der Pose im Werk STILLNESS wegführen würde. Dennoch muss erwähnt werden, dass die Klassifizierung des Films als Medium der Zeit

6o Achim Hochdörfer, »Tacita Dean Makes a Film«, in: Tacita Dean. seven books grey, Bd. 7: Essays über die Arbeiten von Tacita Dean, hg. v. Museum für Moderne Kunst Stiftung Ludwig Wien, Steidl Publishers, Göttingen 2011, S. 39-43, S. 41.

61 Vgl. Deleuze, Das Bewegungs-Bild, S. 211 f. 
vor allem den oben bereits erwähnten strukturellen Film geprägt hat, die neben dem Expanded Cinema bis heute vor allem im Kunstbereich einflussreichste Filmströmung. Deren Hauptvertreter/innen strebten eine gezielte Abkehr vom poetischen, malerischen Experimentalfilmkino an und widmeten sich vor allem der Dimensionen der Zeitlichkeit ihres filmischen Mediums:

Important to the presentation of process is an attention to temporality, as time is film's primary dimension (I would say material), and attention to duration (how long something lasts). It is usual in this connection to begin by adducing the exposition of the possible one to one relationship between shooting time and reading time, adducing an equivalence between the duration of the event recorded and the duration of the film representation of that event. ${ }^{62}$

Wie Malcolm LeGrice hier in Bezug auf die duration pieces von Andy Warhol aus den 196oer Jahren verdeutlicht, wird die Zeit als das Material des Filmemachers aufgefasst - ihre Dauer beziehungsweise ihre Wahrnehmung durch die Zuschauer/innen, das Verhältnis zwischen der Zeit der Aufnahme, der Filmzeit und der Realzeit während der Rezeption hat Künstler und Filmemacher wie Warhol, Michael Snow, George Landow, Peter Kubelka, Birgit Hein, Ken Jacobs, Joyce Wieland und viele andere im Kern interessiert. ${ }^{63}$ Tacita Dean knüpft einerseits an diese Vorläufer an, wenn sie die Spulenlänge, das Filmkorn und die haptischen Vorgänge der Montage als essenziell für ihre Filme bezeichnet. Andererseits sind in ihren Arbeiten die Erzählung, die Bildgestaltung, das Motiv der Natur und Porträts bestimmter Personen zu präsent, als dass sie auf ebenjene medialen und materiellen Aspekte der Werkgenese reduziert werden könnten.

\section{Die Posen Merce Cunninghams}

Tacita Deans Arbeit verbindet zwei Besonderheiten der Pose miteinander: Die Einnahme der Pose durch einen Tänzer, dessen Körperlichkeit auch in absoluter Bewegungslosigkeit einen tänzerischen Ausdruck hat und dessen Performance die verschiedenen zeitlichen Dimensionen der Pose, wie sie oben erläutert wurden, verdeutlicht; und die Kombination aus Wiederholung und Variabilität, welche die Pose als Figur selbst, aber darüber hinaus auch den Aufbau der Installation Merce Cunningham performs STILLNESS kennzeichnet. Die Kombination dieser beiden zunächst getrennt erscheinenden Bereiche - hier die dominante Körperlichkeit Cunninghams und sein

62 Malcolm LeGrice, After Image 7, London 1978, S. 121, zit. n. Gidal, Materialist Film, S. 2.

63 Zum strukturellen Film siehe die Literaturhinweise in Kapitel 2, Fußnote 69. 
Oszillieren zwischen Bewegung und Innehalten, dort die psychoanalytischhistorische Deutung der Pose als theoretische Figur und die Übertragung dessen auf die Filminstallation - zeigt, dass die Verflechtung von sicht- und spürbarer Zeitlichkeit des Bildes und der Historizität der Darstellungsmittel in Deans Werk exemplarisch für andere filmbasierte Arbeiten zum Ausdruck kommt.

In Tacita Deans Filminstallation spielt die Interaktion von Wiederholung und Variabilität eine wichtige Rolle, denn sie betrifft die Wahrnehmung der Installation als Ganzes ebenso wie die Pose, die Cunningham einnimmt, um seine Interpretation von 4'33" aufzuführen. Auf den ersten Blick erscheint es für uninformierte Betrachter/innen, als sähen sie sechs Mal denselben Film auf verschiedenen Leinwänden. Dann erkennen sie die unterschiedlichen Blickwinkel auf Cunningham, die andere Blicke in den Raum ermöglichen, sowie die singuläre Aufnahme seines Kopfes. Erst nach und nach stellt sich die Erkenntnis ein, dass auch hier nicht eine einzelne Performance aus verschiedenen Perspektiven gefilmt wurde, sondern dass es sich um sechs voneinander getrennte Aktionen und Aufnahmen handelt (dies ist natürlich auch dem Untertitel zu entnehmen - six performances; six films). Jede >Aufführung; unterscheidet sich von der anderen, so, wie sich stets auch der filmische Blick auf Cunningham ändert. Es ist jedoch bei den zeitgleich im Loop laufenden Filmen nicht möglich, eine Reihenfolge zu erkennen, das Original und die Wiederholungen auszumachen. So, wie eine eingenommene Pose nie eine exakte Kopie ist, sondern durch die ihr eigene Originalität und die zwangsläufigen Abweichungen vom Ursprung, die die Grenzen zwischen Kopie und vermeintlichem Original verwischen, stets eine Interpretation beziehungsweise ein Zitat darstellt, so ist die Pose auch hier auf zweifache Weise zu denken: Einerseits wiederholt der gefilmte Tänzer seine Haltungen und ihre genau getimten Veränderungen, die Abweichungen sind jedoch so minimal, dass nur im anschaulichen Vergleich, im Hin- und Herblicken zwischen den Filmen, Differenzen erkannt werden können. Andererseits richtet die Künstlerin den Sucher ihrer Kamera wieder und wieder auf Cunningham, sie umkreist ihn und offenbart von verschiedenen Standorten aus jeweils einen anderen Blick auf ihn; es gibt dabei jedoch weder die eine Ansicht, die wie die Schauseite einer Skulptur alle wichtigen Aspekte zeigt, noch entsteht durch Addition der Filme ein vollständiges Bild des Geschehens. Dean schreibt dazu:

Jede Vorführung scheint unzählige Unterschiede aufzuweisen, selbst in der Wiederholung. In einem sitzt er wie Whistlers Mutter, ein lebendes Abbild im Bildfeld des Films. In einer anderen starrt er uns mit dem von wilden Locken 
umrahmten Haupt eines griechischen Gottes durch die Schlieren und Fingerabdrücke auf dem Spiegel entgegen. ${ }^{64}$

Aus dem Zitat wird deutlich, dass die Künstlerin selbst nicht umhin kann, die wie zufällig eingenommene Haltung Cunninghams im Kontext kunsthistorischen Bildwissens zu reflektieren. Ihre Vergleiche mit Whistler und mythologischen Darstellungen weisen den Bildern des Tänzers wie von selbst den Status von Repräsentationen zu, den diese jedoch nicht erst durch den Sucher der Kamera, sondern (mit Owens) bereits als selbstreflexive Geste des Posierens erhalten. Insbesondere bei Cunningham als Tänzer und Performer, der seinen Körper als darstellendes Material betrachtet, kann vorausgesetzt werden, dass er die Dynamik dieser Blickbeziehungen und deren Auswirkungen kennt, ja, verinnerlicht hat. Hierauf soll im nächsten Abschnitt näher eingegangen werden.

\section{Merce Cunningham performs STILLNESS im Rahmen von Cunninghams Tanzästhetik und Technik}

Bereits der Titel der hier besprochenen Filminstallation Merce Cunningham performs STILLNESS (in three movements) to John Cage's composition 4'33" with Trevor Carlson, New York City, 28 April 2007 (six performances; six films) enthält drei Schlüsselwörter für die Interpretation der Arbeit im Hinblick auf Cunninghams Tanzästhetik: »performs«, »stillness « und »movements«. Oben wurde bereits angedeutet, dass die Bewegung in Zeit und Raum zentral für Cunninghams Tanzästhetik ist. Wie Sabine Huschka ausführt, entwickelt Cunningham jedes Stück aus einer Anfangsbewegung heraus, die weiter gestaltet wird, ohne jedoch dem Diktum des expressiven Ausdrucks des Modern Dance unterworfen zu sein: »In Cunninghams Tanzstil dagegen bricht das einmal gefundene momentum, den ganzen Körper in einen Bewegungsfluß umgreifend, kurz darauf zu einem neuen ab. Die Bewegungen bahnen sich jäh und dennoch sanft eine neue Richtung und finden zu neuen Zeiten.« ${ }^{65}$ Sein Tanz zeige »bewegte, sich bewegende Körper «, ${ }^{66}$ vermeide aber eine psychische Ausdeutung und eine Verortung innerhalb einer Erzählung. Es gehe jedoch in Cunninghams Tanzästhetik trotz seiner konzeptionellen Ansicht über den Tanz als Bewegung in Zeit und Raum nicht um eine reine Bewegung, die von der Bedeutung der Körperlichkeit des Tänzers losgelöst ist.

64 Tacita Dean, Informationsblatt zur Ausstellung anlässlich des Kurt-Schwitters-Preises 2009 im Sprengel Museum Hannover, 2010, nicht paginiert.

65 Huschka, Merce Cunningham und der Moderne Tanz, S. 324.

66 Ebd., S. 208. 
Die Bewegungen von Cunninghams Tänzern sind, wie Huschka herausarbeitet, entgegen der zeitgenössischen Rezeption nicht >rein< oder >abstrakt<, sondern als »abstrakt lässt sich ein Tanz somit nur in Bezug auf seine Kompositionsverfahren bezeichnen, die [...] Bewegungen nach dynamischen, rhythmischen oder räumlichen Ausdruckswerten stilisieren « ${ }^{67}$

Man könnte an dieser Stelle einwenden, dass die Zuschauenden in Deans Filminstallation überhaupt keinen Tanz sehen. Im Gegenteil, es findet fast gar keine Bewegung statt, vom bereits beschriebenen Zittern und der sporadischen Gewichtsverlagerung und Bewegung der Arme abgesehen. Trotzdem muss Cunninghams Performance unter dem Vorzeichen seiner Tanzästhetik betrachtet werden, denn sie ist eine, wenn auch auf das Wesentliche reduzierte, Choreografie. Wenn Tanz als Bewegung in Zeit und Raum charakterisiert wird, ist die Abwesenheit von Bewegung, das Halten einer Pose, ebenso eine tänzerische Figur, nämlich die bewusste Entscheidung des Innehaltens für eine bestimmte Zeit im Raum. Cunninghams Verständnis von Tanz, seine Einschätzung von Bewegung, des Körpers des Tänzers / der Tänzerin und seine choreografische Arbeit mit Rhythmus und zeitlicher Struktur beeinflussen sein ganzes Dasein: seine im Film im Verfall gezeigte Körperlichkeit, seine Erscheinung, sein Blick und seine Haltung, aber auch Cunningham als historische Person mit seiner persönlichen Bindung an die Kunst der Avantgarde und als Erneuerer und Provokateur, der den Modern Dance stark prägte. Ohne Einbeziehung der Historizität seiner Person würde der Interpretation von Deans Arbeit ein entscheidendes Puzzleteil fehlen.

Cunningham hat sich in seinem Tanzstil mit den elementaren Fragen der menschlichen Bewegung beschäftigt: Wann ist ein Körper in Ruhe, wann ist er gespannt? Wie ist das Gewicht beim Liegen, Sitzen, Stehen verteilt, wie wird Balance, wie Instabilität erreicht? Welche Bewegungen können miteinander kombiniert werden? In Merce Cunningham performs STILLNESS zeigt sich Cunningham als Mann, der an seinen körperlichen Grenzen angelangt ist. Er sitzt auf einem Stuhl, dies jedoch nicht indifferent, sondern isoliert in der Mitte des Raumes, der noch die Spuren des Trainings trägt, und er zeigt seinen Körper als das, was er ist: ein dysfunktionales Arbeitsgerät des Tänzers, Zeichen dafür, dass der Verlust an Beweglichkeit auch an ihm nicht vorbeigegangen ist, Symbol des Alters, aber auch trotziger Widerspruch eines geistig hellwachen Menschen. ${ }^{68}$ Tacita Dean beschreibt: $»$ Trevor Carlson, the company director,

67 Ebd., S. 278.

68 Huschka beschreibt, wie der gebrechliche Cunningham, der mühsam und gebeugt ging, sich, sobald er tanzte, leichtfüßig und mit kleinen, exakten Schritten über die Bühne bewegte. Vgl. ebd., S. 12. 
signaled the last five seconds by putting up his hand for Merce to see. Like a bird of prey, Merce perceived this without gesture, broke his pose and then resettled for the next movement. «69

Die zeitliche Strukturierung von Bewegung und die gleichzeitige Strukturierung von Zeit durch die ausgeführte Bewegung spielt in Merce Cunninghams Tanzästhetik eine große Rolle. Da seine Choreografien sich nicht an der Musik orientieren, in den meisten Fällen sogar ganz ohne musikalische Begleitung auskommen, sind sie »unmittelbar mit der Notwendigkeit konfrontiert, die Zeiten ihrer Bewegungen fortan metrisch andersweitig (sic!) zu regulieren « ${ }^{70}$ Das heißt, die Zeit wird für den Choreografen zu einem »frei verfügbaren Parameter « und die Rhythmisierung der Bewegungen der Tänzer/innen beruht auf zufällig festgelegten Zeiteinheiten, welche von den Tänzer/innen im Training verinnerlicht werden. Die zeitliche Struktur, führt Cunningham $195^{2}$ aus, verschafft dem Tanz unabhängig von Musik große Freiheit:

[B] ut if one can think of the structure as a space of time in which anything can happen in any sequence of movement event, and any length of stillness can take place, then the counting is an aid towards freedom, rather than a discipline towards mechanization. A use of time-structure also frees the music into space, making the connection between the dance and the music one of individual autonomy connected at structural points. ${ }^{71}$

Deutlich ist hier zu beobachten, wie sehr Cunninghams Arbeit mit der der Aleatorik in John Cages Musik korrespondiert und wie Merce Cunningham performs STILLNESS diese Korrespondenz vor Augen führt. Die zufällig erreichten Zeitabschnitte, welche Trevor Carlson mittels Stoppuhr anzählt, geben den zufälligen Geräuschen als Musik Raum und fungieren als Gerüst der Choreografie, sie gliedern die Arbeit und machen die nächsten Schritte vorhersehbar, wenngleich das tatsächliche Geschehen innerhalb der Zeitspanne unvorhersehbar bleibt. So erreicht Cunningham durch die zeitliche Struktur ein Maximum an Freiheit, die Dauer des Zeitabschnitts für die Betrachterinnen

69 Tacita Dean, »Merce Cunningham performs >Stillness< (in three movements) to John Cage's Composition >4'33"< with Trevor Carlson, New York City, 28 April 2007 (six performances; six films), 2008«, in: Tacita Dean. seven books grey, Bd. 1: Film Works with Merce Cunningham, hg. v. Museum für Moderne Kunst Stiftung Ludwig Wien, Steidl Publishers, Göttingen 2011, S. 3-21, S. 5 .

70 Huschka, Merce Cunningham und der Moderne Tanz, S. 366.

71 Merce Cunningham, »Space, Time and Dance« (1952), in: Richard Kostelanetz (Hg.), Merce Cunningham. Dancing in Space and Time, Pennington, NJ 1992, S. 37-39, S. 39. 
der Arbeit auch durch Bewegungslosigkeit erlebbar und fühlbar zu machen. Wie bei dem Musikstück 4'33" bilden die Hintergrundgeräusche der Straßen Manhattans, welche über den synchronisierten Ton mit aufgenommen wurden, die zufällige $>$ Musik .

Stillness ist bei Cunningham nicht mit Stille als Gegensatz zum Geräusch gleichzusetzen, sondern als Gegenpart zur Bewegung im Tanz. ${ }^{72}$ Dass sich Bewegung und Nicht-Bewegung nicht ausschließen, nicht zwei Seiten einer Medaille sind, sondern als notwendige Zustände einer raumzeitlichen Orientierung, ähnlich dem Kippmoment des Pendels, zusammengehören, ist ein grundsätzliches Kennzeichen der Pose, die eben ein Innehalten, aber kein finales Arretieren der Bewegung markiert. Für Brandstetter besteht das paradoxe Verhältnis der Pause zur Bewegung darin, dass jene »immer schon zur Gesamtheit der Bewegung - zur Bewegungsfiguration - gehört; dass mithin das Anhalten bzw. das Stillstellen der Bewegung die Bedingung der Möglichkeit von Bewegungsausdruck « ist. ${ }^{73}$ Erwähnenswert ist hier auch die Doppeldeutigkeit im Titel: STILLNESS (in three movements) bedeutet korrekt übersetzt >in drei Sätzen<, in seiner anderen Bedeutung als >in drei Bewegungen $<$ bezeichnet der Titel jedoch auch die Darstellung des Einen durch sein Anderes, der NichtBewegung durch Bewegung.

Wie verhalten sich nun Tacita Deans Kameraarbeit und die Präsentation der Filme zu den Performances und deren Deutung innerhalb von Merce Cunninghams Tanzästhetik? In den drei hier aufeinandertreffenden ästhetischen Ausdrucksweisen, dem Tanz, der Musik und dem Film, spielt der Umgang mit der Zeit eine große Rolle. Die obige Beschreibung der Ästhetik der Stillstellung in Tacita Deans Filmen zeigt, dass Dean Zeit als zentrales Charakteristikum des filmischen Mediums betrachtet: »The time in my films is the time of film itself.« ${ }^{74}$ Für sie ist von elementarer Bedeutung, dass sich die filmische Zeit in ihrem physischen Äquivalent des Filmstreifens materiell fassen lässt, und zwar von der manuell mit dem Material arbeitenden Künstlerin ebenso wie von den Zuschauer/innen, die den Transport des Films im Projektor mitverfolgen können, wenn sie im Ausstellungsraum stehen. ${ }^{75}$

72 Vgl. Schröder, »So that one becomes aware of the presence of a sound - or it's absence«", S. 65 .

73 Brandstetter, »Pose - Posa - Posing «, S. 46.

74 Tacita Dean, »Film«, in: Nicholas Cullinan (Hg.), Tacita Dean. Film, Ausstellungskatalog Tate Modern, London 2011/2012, London 2011, S. 15-33, S. 19.

75 Die Positionierung der Projektoren im Raum ist nicht bei jeder Arbeit gegeben, was ebenso den räumlichen Gegebenheiten wie den Aufsichtsmöglichkeiten geschuldet sein mag. 
In ihrer Arbeit Magnetics werden die Zelluloidstreifen selbst ausgestellt, sie stehen für die Linearität des Films und die physische Verkörperung der Zeit im Film. ${ }^{76}$ In Merce Cunningham performs STILLNESS entspricht die jeweilige Filmzeit der Screenzeit und hat somit auch ihre analoge Entsprechung in der Spule; Dean verzichtet hier auf Schnitte und Montage, wie sie zur Erzeugung eines filmischen Raum-Zeit-Kontinuums eingesetzt werden, und reproduziert das während der Performance herrschende Raum-Zeit-Kontinuum, um über den Film ein analoges Zeitverständnis im Sinne einer Gleichsetzung von realer Zeit, Filmzeit und erlebter Zeit zu vermitteln. Die Montage findet jedoch auf einer anderen Ebene, nämlich der des Ausstellungsraums, statt. Hier werden durch die asynchrone Schaltung der einzelnen Filme verschiedene >Zeitfenster übereinandergelegt, sie werden nicht linear nacheinander, sondern parallel präsentiert, wie in einer Art begehbarem Cluster. Die Schnittstelle der unterschiedlichen Blickrichtungen auf die Posen von Merce Cunningham ist der Zuschauer oder die Zuschauerin. Diese Präsentationsform ist insofern interessant, als sie der Linearität der einzelnen Filme entgegensteht und die einzelnen Performances, die nacheinander aufgenommen wurden, zueinander in Beziehung setzt. Aus den verschiedenen Blickwinkeln auf Cunningham setzt sich so nur von der Position des bewegten Betrachters oder der Betrachterin aus ein Bild der Performances in zeitlicher >Schichtung und >Überlappungく zusammen. Die Zusammenführung verschiedener räumlicher und zeitlicher Blickwinkel auf Cunningham innerhalb der simultanen Wahrnehmung der Leinwände ermöglicht im Gegensatz zur Rezeption im Kino oder einer Blackbox mit Stuhlreihen eine »Individualisierung der Filmerfahrung « ${ }^{77}$ Dieser Aspekt unterscheidet die Betrachtung und Wahrnehmung des Films stärker noch als die vermeintliche Mobilität im Ausstellungsraum von der Filmerfahrung im Kinoraum.

\section{Exkurs: Obsolescence and Ruin-Aspekte der Historizität}

Die hier besprochene Arbeit gehört in eine Reihe von Werken, die nicht zusammenhängend entstanden, jedoch trotzdem einen eigenen Komplex innerhalb von Deans OEuvre bilden. Es handelt sich um Porträts hauptsächlich älterer Männer, in der Mehrzahl Künstler. Dazu gehören Filme über die bildenden Künstler Mario Merz, Cy Twombly und Claes Oldenburg, über den Autor und Übersetzer Michael Hamburger sowie zwei Filme über Merce

76 Theodora Vischer, »The Story of Linear Confidence«, in: dies., Isabel Friedli (Hg.), Tacita Dean. Analogue, Zeichnungen 1991-2006, Ausstellungskatalog Schaulager, Basel 2006, Göttingen 2006, S. 11-29, S. 29, Anm. 31.

77 Rebentisch, Ästhetik der Installation, S. 191. 
Cunningham, die hier besprochene Installation und der Film Craneway Event von 2009, in dem Dean den Proben zu einer der letzten Aufführungen unter Cunningham beiwohnt. ${ }^{78} \gg I$ have ended up filming people because I want to trap something of them on this earth before things change. «79 In diesem Satz zeigt sich eine wichtige Motivation für Deans Arbeiten, nämlich der Kampf gegen das Vergessen durch analoge Aufzeichnung auf Film. So wie die Performance von Cunningham - eine der letzten selbst aufgeführten Arbeiten des Tänzers - eine Hommage an Cage ist, indem Cunningham dessen wohl wichtigste Komposition in sein Medium, den Tanz, überträgt (und $4^{\prime} 33^{\prime \prime}$ im wahrsten Sinne des Wortes verkörpert), so ist Merce Cunningham performs STILLNESS eine Hommage an Cunningham selbst, die seine Arbeit aufzeichnet, dokumentiert und für die Nachwelt festhält - »before things change«, als hätte Dean den nahen Tod des Tänzers vorhergesehen. Die Erinnerung an Vergangenes und gleichzeitige Mahnung an das Publikum, sich der eigenen begrenzten Lebenszeit bewusst zu sein, ist ein klassisches Thema der Kunstgeschichte in Memento-Mori-Darstellungen. Insofern ist die Bezeichnung der Arbeit als ein Memento Mori für Cunningham treffend, gerade auch mit Blick auf Trevor Carlson, der im Hintergrund immer wieder die ablaufende Zeit für die jeweilige Haltung und den Countdown für die nächste Pose anzählt. ${ }^{80}$ Das Alter von Dingen und seine sichtbaren Spuren beispielsweise an Bauwerken, aber eben auch an Menschen, sind in Deans Arbeiten stets präsent. Unter dem Stichwort der »obsolescence« beschreibt sie ihre Leidenschaft für Dinge, die überaltert sind, einer anderen Zeit entstammen und kurz vor dem Verschwinden stehen. ${ }^{81}$ Die Zeitlichkeit solcher Dinge sieht sie in ihrer Arbeit mit analogem 16-mm-Film gespiegelt und verdichtet - einem Medium, dessen materieller Schönheit sie in Kodak (2006) ein Denkmal gesetzt hat.

Die amerikanische Kunsthistorikerin Christine Ross hat in ihrer Analyse von Merce Cunningham performs STILLNESS zwei Aspekte der Arbeit, die die Historizität betreffen, unter dem Begriff der »ruiniste tradition«, der Ruinenästhetik, diskutiert. ${ }^{82}$ Unter diesem Begriff werden in der Kulturwissenschaft

78 Mario Merz, 2002; Michael Hamburger, 2007; Merce Cunningham performs STILLNESS (in three movements), 2008; Craneway Event, 2009; Manhattan Mouse Museum, 2011; Edwin Parker, 2011. Des Weiteren können die Filme Boots und Salvation Sisters zu Deans >Porträts< gezählt werden.

79 Tacita Dean in Carvajal, $»$ Film is a Medium of Time«, S. 6o.

8o Dieter Daniels, Inke Arns, »Sounds Like Silence«, in: dies., Sounds like silence, S. II-IV (deutschsprachiges Beiheft).

81 Tacita Dean, »Artist Questionnaire. 21 Responses«, in: October 10o, Frühjahr 2002, S. 6-97, S. $26 \mathrm{f}$.

82 Vgl. Christine Ross, The Past is the Present; It's the Future, Too, New York / London 2012, Kapitel 4: »Ruination Gone Wrong«. 
die Wertschätzung von Architektur im Verfall und die semantische Neubesetzung von Ruinen vor allem seit der Romantik gefasst. ${ }^{83}$ Ross sieht die Ruinenästhetik als spezifisches Thema von Deans Schaffen: zum einen in der Darstellung von verschwindenden, verfallenden Dingen, zum anderen in der Verwendung veralteter Technik, die durch Abnutzung zu ihrem eigenen Verschwinden beiträgt. Film würde dadurch in Deans Kunst als Medium des Verlaufs der Zeit offenkundig. ${ }^{84}$ Ross geht nicht näher auf den historischen Diskurs über die Ästhetisierung des Verfalls ein, verortet jedoch Tacita Deans Arbeit der Darstellung ebenso wie der Materialität nach in direkter Tradition der Ruinenästhetik: »The ruiniste procedure is doubly active in the filmworks' recording of ruins (which belongs to the contingency of cinematic film) and the filmworks' display as a ruin and its own ruining of the celluloid (which discloses the rationalization of cinematic time).«85 Die Ruine wird für sie zum Schlüsselbegriff, um einerseits den alternden Körper von Merce Cunningham, andererseits den stetigen Verfall des Filmmaterials und die Veralterung der Technik zu charakterisieren. Ross beschreibt die Wertschätzung von Ruinen als spezifisch modernes Symptom, das sich seit der Romantik als Kontrast zum Zukunftsglauben der Moderne notwendigerweise entwickelt habe, und sie stellt fest, dass das Bild der Ruine daher ein inhärenter Bestandteil der Moderne sei: »[R]uinisme is a critical voice of modernity within modernity. $[\ldots][\mathrm{T}]$ he imaginary of ruins is an inherent constituent of modernity itself. ${ }^{86}$ Bei Dean werde die Ruine als Bild jedoch dadurch noch übersteigert, dass der Film sich selbst im Zuge seiner materiellen Präsentation ruiniere, durch Abnutzung auflöse. Dass die Ruine in Deans Werk als Symbol der Historizität der Moderne eine Rolle spielt, stellte bereits Erica Balsom fest, wobei sie sich jedoch auf die architektonischen Filme Deans bezog. ${ }^{87}$ Wie Ross identifiziert sie die Faszination der Ruine als Symptom der Moderne, bezieht jedoch die Vorliebe Deans und anderer Künstler für analogen Film auf eine neu entstehende Auratisierung des Films im Kunstkontext.

83 Vgl. Hartmut Böhme, »Die Ästhetik der Ruinen«, in: Dietmar Kamper, Christoph Wulf (Hg.), Der Schein des Schönen, Göttingen 1989, S. 287-304.

84 »It [the filmic image, N. S.] is explored to overdetermine film as a medium of temporal passing insofar as ruining surfaces as a manifestation of the passage of time are not only filmed but are also what occurs to film« (Ross, The Past is the Present; It's the Future, Too, S. 191).

85 Ebd., S. 173 f.

86 Ebd., S. 195 .

87 Erika Balsom, »A Cinema in the Gallery, a Cinema in Ruins«, in: Screen 5o/4, Winter 2009, S. $411-427$, S. 421. 
Interessant ist bei beiden, dass sie die Materialität der Filmapparate und ihre Historizität unter dem Stichwort der Ruinen behandeln und so die Zeitlichkeit des Bildgeschehens und die Historizität der verwendeten Technik zueinander in Bezug setzten. Wenn die architektonische Metapher auch nicht in allen Punkten stimmig zu sein scheint, transportiert sie jedoch ein Gefühl der Melancholie und Nostalgie für die Ästhetik des Verschwindens, das bei Deans Einstellung zum Film und zu ihren Motiven stets mitschwingt.

Noch wichtiger als die Übertragung des architektonischen Begriffs auf den Körper des Tänzers und die Technik des Films erscheint jedoch Ross' Beschreibung der bildlichen Strukturen innerhalb des Bildrahmens und deren Wiedergabe über das Zelluloid als »media skin «, 88 eine Art mediale Haut. Die verschiedenen Oberflächen - einerseits des Körpers von Cunningham, andererseits die ihr Alter zeigenden Wände und Spiegel des Studios - machten im zitternden Bildeindruck des Films als »temporizing volume ${ }^{89}$ die Zeit selbst sichtbar. »Temporizing volume« ist nach Ross' Definition »a volume generated by the surface/depth, stasis/movement interactions between the recorded bodies and the celluloid strip as well as between the spectator and the screen $\kappa^{90}$ Die Einstellungen, die einen Blick auf Cunninghams faltige Haut erlauben, verflechten die minimalen unbewussten Bewegungen des Körpers mit dem Zittern des kinematografischen Bildes und halten dabei doch eine Spannung, die die »media skin as a stilled site of micro changes $~^{91}$ ausweisen: »In Dean's installation, the stilled media skin is the modality by which temporal passing is conveyed; stillness is the procedure that enables the skin to act as a manifestation of temporality.« 92

Eine ähnliche Interaktion, welche Ross als »media skin« beschreibt, geschieht in der Pose: Deren Zeitlichkeit als innegehaltene Bewegung setzt die Figuration des Körpers in der Figur (Pose) zur filmischen Dauer der Einstellung und der Mikrobewegung des filmischen Bildes in Beziehung. Der Körper stellt sich in der Pose selbst still und bildet für das immer leicht bewegte Bild des analogen Films die Fläche, als Film sichtbar zu werden. Die latenten Bewegungen, die in jeder gehaltenen Pose aufscheinen, korrespondieren mit der Stillstellung, die einerseits Tacita Deans Filmtechnik, andererseits der Pose des gefilmten Subjekts unter dem Blick der Kamera eigen ist. Die Arbeit offenbart in ihrer Einfachheit, so wie viele Werke Deans, ein Kaleidoskop

\footnotetext{
88 Ross, The Past is the Present; It's the Future, Too, S. $182 \mathrm{ff}$.

89 Ebd.

$90 \quad$ Ebd., S. 186.

91 Ebd., S. 183.

92 Ebd., S. 186.
} 
unterschiedlicher Zeitlichkeiten, deren Beobachtung wiederum Zeit erfordert und den Betrachter oder die Betrachterin selbst dazu verleitet, vor der Leinwand in Stillstellung zu verharren.

\section{Performance und Performativität}

Merce Cunningham performs STILLNESS ist bisher als sechsteilige Installation, wie sie in der Dia Art Foundation sowie im Sprengel Museum Hannover zu sehen war, analysiert worden. Ursprünglich jedoch war sie als Teil eines Events geplant, welches das Format Ausstellung mit zeitbasierten Arbeiten in den Raum des Theaters übertrug: Il Tempo del Postino, eine Gruppenausstellung im Manchester Opera House vom 12. bis 14. Juli 2007 anlässlich des Manchester International Festival. An der Ausstellung nahmen neben Tacita Dean zahlreiche namhafte Künstler wie Douglas Gordon, Anri Sala und Pierre Huyghe teil, sie wurde von Hans Ulrich Obrist und Philipp Parreno kuratiert. Tacita Dean hatte, wie sie in einem Interview berichtet, die Arbeit mit Merce Cunningham als stillen Gegenpol zu den anderen zu erwartenden Arbeiten geplant und ursprünglich mit Marcel Marceau, dem berühmten Pantomimekünstler, arbeiten wollen. ${ }^{93}$ Da dieser jedoch erkrankt war, entstand die Idee, Merce Cunningham zu 4'33" performen zu lassen. Il Tempo del Postino fand auf einer Theaterbühne statt, und dort wurde der erste der Filme auf einer am Boden stehenden Leinwand aufgeführt. Diese wurde von hinten mit dem Film bespielt, damit der Eindruck entstand, als säße Merce Cunningham tatsächlich auf der Bühne und agierte live für das Publikum. ${ }^{94}$ Im Nachhinein entwickelte Dean die sechs entstandenen Filme zu der raumfüllenden Installation weiter. Diese Umstände der Entstehung von Merce Cunningham performs STILLNESS richten den Blick auf einen wichtigen Aspekt dieser Arbeit wie auch der vorhergehenden Werke von Coleman, der abschließend diskutiert werden soll, nämlich den Zusammenhang von Performance, Performativität und Film. Die Posen als nichtsprachliche, körperliche Äußerungen werden hier in Bezug zu den Bedingungen ihrer Sichtbarkeit als performativ beschrieben und die Verknüpfung der Geschichtlichkeit der Werke mit dem Ereignis ihrer Aufführung, wie Mieke Bal und Dorothea von Hantelmann sie hervorheben, skizziert.

93 Tacita Dean im Gespräch mit Hans Ulrich Obrist, »A Conversation at the Hochschule für bildende Künste Hamburg, June 2010«, in: Hans Ulrich Obrist (Hg.), Tacita Dean. The Conversation Series 28, Köln 2012, S. 51-78, S. 63 .

94 Tacita Dean im Gespräch mit Hans Ulrich Obrist, »Il Tempo del Postino, At the Manchester Opera House stage door, 2007 «, in: Hans Ulrich Obrist (Hg.), Tacita Dean. The Conversation Series 28, Köln 2012, S. 45-50, S. 48. 
Wenn Gabriele Brandstetter im obigen Zitat die Pose als Mittlerin zwischen »Performance und Performativität « beschreibt, erscheint dies zunächst als naheliegende Engführung, suggeriert doch der gleichlautende Wortstamm >perform< eine inhaltliche Nähe beider Konzepte zueinander. Es ist jedoch wichtig, hier zu differenzieren, da die Begriffe Performance, Performanz und Performativität weder im englischsprachigen noch im deutschsprachigen Diskurs einheitlich verwendet werden. Wird im hier zitierten Kontext unter Performance ein aufgeführtes, als Werk verstandenes künstlerisches Ereignis im Rahmen der Performance Art verstanden, so verbirgt sich hinter der Performativität ein ursprünglich linguistisches Konzept, das auf die Kulturwissenschaften übertragen wurde. Dass Performanz und Performativität sich nicht wie der »Objektbegriff zu seinem theoretischen Begriff« verhalten, sondern zu jeweils anderen Ordnungen gehören, stellt Mieke Bal zu Beginn ihres Aufsatzes »Perfomativität und Performanz« dar. ${ }^{95}$ Performanz sei »die einzigartige Ausführung eines Werks« und Performativität »jener Aspekt eines Wortes, der tut, was er sagt [...]. Obwohl beide Worte vom selben Verb >to perform < abgeleitet sind, sind sie als Konzepte nicht verbunden. ${ }^{96}$ Der Ausdruck >performativ wurde von dem Sprachwissenschaftler John L. Austin geprägt und bezeichnet die Eigenschaft mancher sprachlicher Äußerungen, nicht nur einen Sachverhalt zu benennen, sondern mit der Äußerung zugleich eine Handlung zu vollziehen. In seiner Vorlesung How To Do Things With Words von 1955, die 1972 unter dem Titel Zur Theorie der Sprechakte auf Deutsch erschien, ${ }^{97}$ beschreibt Austin beispielsweise die Vermählung (»Hiermit erkläre ich Sie zu Mann und Frau «) oder die Schiffstaufe als solche Sprechakte, die neue Tatsachen schaffen: »Denn die Sätze sagen nicht nur etwas, sondern sie vollziehen genau die Handlung, von der sie sprechen. Das heißt, sie sind selbstreferentiell, insofern sie das bedeuten, was sie tun, und sie sind wirklichkeitskonstituierend, indem sie die soziale Wirklichkeit herstellen, von der sie sprechen $\ll, 98$ fasst Erika Fischer-Lichte zusammen. Die Bedeutung eines Sprechakts hängt demnach von seinem Gelingen ab, »performative Äußerungen haben keinen

95 Mieke Bal, »Performativität und Performanz«, in: Jörg Huber (Hg.), Kultur-Analysen, Zürich 2001, 197-241. Der Übersetzer weist darauf hin, dass im englischen Original von performativity und performance die Rede sei, wobei Letztere mit dem im Deutschen unüblichen Performanz übersetzt wird, wohl auch, um eine Abgrenzung zur Kunstform der Performance vorzunehmen.

96 Ebd., S. 197.

97 John L. Austin, How to do Things with Words, London/Oxford 1975; John L. Austin, Zur Theorie der Sprechakte, Stuttgart 1979.

98 Erika Fischer-Lichte, Ästhetik des Performativen, Frankfurt am Main 2004, S. 32. 
logisch-semantischen Wahrheitsbezug «, ${ }^{99}$ wodurch sich die sprachphilosophischen Kriterien der Bedeutungszuweisung ändern. ${ }^{100}$ Für die hier zur Diskussion stehende Frage nach dem Zusammenhang von Performance und Performativität ist, wie Wirth zusammenfasst, die Ausweitung des Begriffs der Performanz wichtig, nämlich

von einem terminus technicus der Sprechakttheorie zu einem umbrella term der Kulturwissenschaften [...], wobei die Frage nach den sfunktionalen Gelingensbedingungen< der Sprechakte von der Frage nach ihren >phänomenalen Verkörperungsbedingungen $<$ abgelöst wurde. ${ }^{101}$

In ihrer Studie Ästhetik des Performativen wendet Fischer-Lichte die Eigenschaft sprachlicher Akte, eine faktische Handlung zu vollziehen, auf den Vollzug körperlicher Handlungen an und bezieht sich dabei auf die von ihr beobachtete "performative Wende« seit den 196oer Jahren. Darunter versteht sie die zunehmende Tendenz von Kunst und Aufführungen - nicht nur in Musik und Theater, sondern auch im Kontext bildender Kunst - zur Ereignishaftigkeit, welche die Zuschauer/innen in die Realisierung des Werks einbezieht. Diese erfordere eine $»$ Rekonzeptualisierung des Begriffs des Performativen « ${ }^{102}$ Austins Konzept der Sprechakte lässt sich indes nicht ohne Weiteres auf körperliche Handlungen übertragen; Fischer-Lichte geht hierfür den Umweg über Judith Butlers Ansatz, Geschlechteridentität als Ergebnis konstituierender Handlungen, von Butler "performativ acts « genannt, zu beschreiben. ${ }^{103} »$ Unter Berufung auf Merleau-Ponty [...] erläutert Butler den Prozeß der performativen Erzeugung von Identität als Prozeß von

99 Uwe Wirth, »Der Performanzbegriff im Spannungsfeld von Illokution, Iteration und Indexikalität«, in: ders. (Hg.), Performanz. Zwischen Sprachphilosophie und Kulturwissenschaften, Frankfurt am Main 2002, S. 9-6o, S. 10.

100 Austin gibt im späteren Verlauf seiner Vorlesung die Unterscheidung zwischen sperformativ< und >konstitutiv< zugunsten der Dreiteilung >lokutionär/>illokutionär//perlokutionär auf, wobei der illokutionäre Akt den performativen Akt ersetzt: Er »vollzieht eine Handlung, >indem man etwas sagt<, im Unterschied zu dem lokutionären Akt, >daß man etwas sagt« (Wirth, »Der Performanzbegriff«, S. 13). Perlokutionäre Akte beziehen sich auf die Wirkung, die beabsichtigten Folgen einer Äußerung, und lokutionär bezeichnet die Phonetik, Grammatik und Semantik eines Satzes.

101 Wirth, »Der Performanzbegriff «, S. 10.

102 Fischer-Lichte, Ästhetik des Performativen, S. 36.

103 Judith Butler, »Performative Acts and Gender Constitution. An Essay in Phenomenology and Feminist Theory«, in: Sue-Ellen Case (Hg.), Performing Feminism. Feminist Critical Theory and Theatre, Baltimore/London 1990, S. 270-282, zit. n. Fischer-Lichte, Ästhetik des Performativen, S. 36 f. 
Verkörperung (embodiment).«104 Durch diese Verkörperung in Form »stilisierter Wiederholung performativer Akte« werde »Identität allererst erzeugt«; die Handlungen sind dabei nie rein individuell, sondern finden immer im Rahmen und auch im Konflikt mit einer Gesellschaft statt. ${ }^{105}$ Diesen Schauplatz verkörpernder Handlungen vergleicht Butler mit einer Theateraufführung, was Fischer-Lichte als Ausgangspunkt nimmt, um eine »Ästhetik des Performativen im Begriff der Aufführung zu fundieren «. ${ }^{106}$

Man kann also feststellen, dass der Vorschlag von Brandstetter, die Pose zwischen der Performativität (im Sinne des ursprünglichen linguistischen Konzepts) und der Performance als Kunstform zu positionieren, einerseits eine problematische Verkürzung aufweist, da hier zwei Kontexte aufgerufen werden, die die Pose als körperliche Ausdrucksform nicht ohne Weiteres ineinander vermitteln kann. Setzt man jedoch voraus, dass körperliche Handlungen im Sinne Butlers wirklichkeitskonstituierend sein können, haben auch künstlerische Ausdrucksweisen weit mehr als nur symbolische Bedeutung. Folgt man Austin und Butler, wird die Prämisse der Diskursivität von Performativität deutlich, die auch für die Pose als zeitliche Figuration eines körperlichen Bildes zutrifft: Nur innerhalb festgelegter und akzeptierter Konventionen können Aussagen, seien sie sprachlicher, körperlicher oder sonstiger Natur, gelingen und kann eine Tatsache geschaffen werden, eine neue Realität sich ereignen. Nur innerhalb festgelegter und akzeptierter Konventionen können also auch Posen und Gesten, so lässt sich dieser Gedanke weiterführen, in ihrer kommunikativen, narrativen, sexualisierten oder sonstigen Funktion gelesen werden und als solche das System, innerhalb dessen sie sich ereignen, widerspiegeln. Die hier besprochenen Arbeiten von Dean ebenso wie von Coleman thematisieren mittels der ästhetischen Figur der Pose die Wiederholung stereotyper Handlungen in Form von Gesten, Attitüden und tableauhaften Körperdarstellungen. Sie stehen in direkter Wechselwirkung mit Regimes der Sichtbarkeit, sei es über die Rolle der fotografischen Kamera, der genrehaften Inszenierung oder des populären Melodrams und seiner Erzählform; bei Dean wird konkret auf die Situation einer Aufführung durch einen Tänzer Bezug genommen. Er verhält sich zur der ihn beobachtenden Kamera jedoch nicht innerhalb der für ihn normalerweise in Anspruch genommenen Repräsentation als Tänzer, vielmehr konstituiert sich Cunningham in seiner direkten Adaption von 4'33" als Medium einer Zeitstruktur, die einen einerseits konkret historischen, andererseits im Kern aleatorischen Ursprung hat.

104 Fischer-Lichte, Ästhetik des Performativen, S. 38.

105 Ebd.

106 Ebd., S. 41. 
Dorothea von Hantelmann hat am Beispiel von Coleman und anderen Künstlern apodiktisch festgestellt, dass Performativität und Performance zwar nichts miteinander zu tun hätten, sich sogar in ihrem Weltbild diametral gegenüberstünden, ${ }^{107}$ dass es aber auch keine nichtperformative Äußerung und gleichermaßen auch kein nichtperformatives Kunstwerk gebe, sondern als Performativität die realitätserzeugende Eigenschaft zu benennen sei, die jeder Äußerung und jedem Kunstwerk innewohne. ${ }^{108}$ Hantelmann sieht in Colemans Werk die Performativität auf der Ebene der Geschichtlichkeit verortet. Sie beschreibt anhand von zwei Arbeiten, Box (ahareturnabout) und GuaIRE, wie sich hier Ereignishaftigkeit und Geschichtsbezug treffen und in der Werkstruktur untrennbar miteinander verbunden sind. Wie bei Austin sei Sagen und Tun aufeinander bezogen - einerseits die Darstellung (von Geschichte) und andererseits ihre ereignishafte Vergegenwärtigung, da jedes Werk Colemans nur in der unmittelbaren Anschauung erfahren werden kann. Welche Rolle die Erinnerung als Mittlerin zwischen Performanz und Performativität spielt, stellt Mieke Bal schließlich am Beispiel von Photograph (1998-1999) vor. In einer virtuosen, weitschweifigen Analyse, auf die hier aus Platzgründen nicht näher eingegangen werden kann, stellt sie heraus, dass das Gedächtnis in seiner identitätsstiftenden und subjektkonstituierenden Funktion ein $A k t$ sei und als solches per se ein kulturelles Gedächtnis. ${ }^{109}$ In seiner Verwicklung mit der Zeit - »das Gedächtnis betrifft die Vergangenheit und geschieht in der Gegenwart «110 - liege sein Potenzial, das Ereignishafte der Performanz mit der realitätsstiftenden Performativität zu verknüpfen. Der Art und Weise, wie in Photograph Stereotypen des Theaters von Jugendlichen in Posen und Gesten adaptiert und überzeichnet würden, gepaart mit Versatzstücken romantischer Dichtung, liege kein simpler Begriff von Aufführung und Theatralität zugrunde, sondern das Bewusstsein, dass das Persönliche immer schon mit dem Politischen verwandt ist. Die »romantische Performanz von Subjektivität«, 111 die hier in der adoleszenten Inszenierung gezeigt werde, ist nach Bal tief im Diskurs der 196oer Jahre um Performancekunst und Theatralität verwurzelt, auf deren Realismusanspruch Coleman kritisch antworte. ${ }^{112}$ Das Gedächtnis hat also die Funktion, über die Vergangenheit und die Geschichte das Ereignishafte mit seiner Handlungsfähigkeit, die nur

\footnotetext{
107 Dorothea von Hantelmann, How to do things with art. Zur Bedeutsamkeit der Performativität von Kunst, Zürich 2007, S. 13 .

108 Ebd., S. $11 \mathrm{f}$.

109 Bal, »Performativität und Performanz«, S. 228.

110 Ebd., S. 204.

111 Ebd., S. 227.

112 Ebd., S. 228.
} 
innerhalb eines kulturellen Rahmens (den das Gedächtnis schafft) wirksam werden kann, zu verbinden.

Beide Autorinnen betonen somit die Geschichtlichkeit und ihre Wechselwirkung mit dem gegenwärtigen Werkereignis als zentral für die Performativität von Kunst. Dass sich dieses Verhältnis, das in der vorliegenden Untersuchung spezifisch für zeitbasierte, bewegte Bilder betrachtet wird, am konkreten Beispiel der Pose zeigen lässt, haben die obigen Betrachtungen der Werke von Coleman und Dean anschaulich gemacht. Dean verlegt mit ihren Filmen die Performances von Cunningham in den öffentlichen Raum der Ausstellung beziehungsweise direkt auf die Theaterbühne, wo performatives Handeln wirksam wird. Durch die Pose, in der die »Figur«, als Resultat des figurativen Prozesses, mit ihrer »Genese «113 verschmilzt, und durch die filmische und räumliche Wiederholung kann sich das musikalische wie das choreografische Werk stets aufs Neue ereignen. Die Posen in Colemans Arbeiten haben eine performative Dimension, die sich nicht nur in der Ereignishaftigkeit des einzelnen Werks vermittelt, sondern in der jeweiligen Aktualisierung des erinnerten Vor-Bildes eingeschrieben ist. In der prozessualen Darstellung des Körpers spiegeln die Posen den Blick des Anderen und werfen die Betrachter/innen so auf die imaginäre Ebene des Bildes, also ihre Beteiligung an der Wirkung des Werks, zurück.

113 Gottfried Boehm, »Die ikonische Figuration«, in: ders., Naef, Müller, Figur und Figuration, S. $33^{-}-52$, S. 34 . 